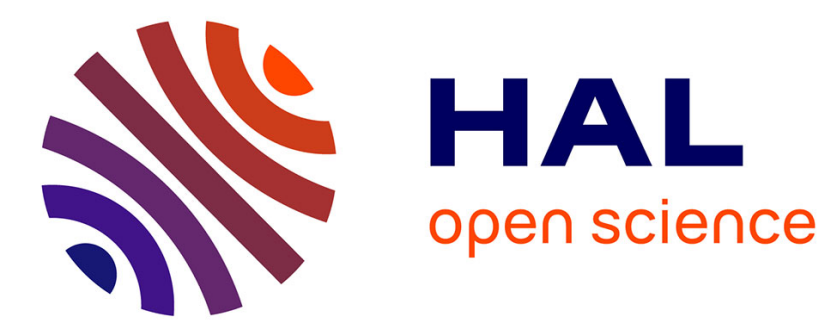

\title{
Effective scaling factors in non-uniform gas radiation modeling
}

Frédéric Andre

\section{To cite this version:}

Frédéric Andre. Effective scaling factors in non-uniform gas radiation modeling. Journal of Quantitative Spectroscopy and Radiative Transfer, 2018, 206, pp.105 - 116. 10.1016/j.jqsrt.2017.10.019 . hal-01934764

\section{HAL Id: hal-01934764 https://hal.science/hal-01934764}

Submitted on 23 Apr 2019

HAL is a multi-disciplinary open access archive for the deposit and dissemination of scientific research documents, whether they are published or not. The documents may come from teaching and research institutions in France or abroad, or from public or private research centers.
L'archive ouverte pluridisciplinaire HAL, est destinée au dépôt et à la diffusion de documents scientifiques de niveau recherche, publiés ou non, émanant des établissements d'enseignement et de recherche français ou étrangers, des laboratoires publics ou privés. 


\title{
EFFECTIVE SCALING FACTORS IN NON-UNIFORM GAS RADIATION MODELING
}

\author{
Frédéric André
}

Univ Lyon, CNRS, INSA-Lyon, Université Claude Bernard Lyon 1, CETHIL UMR5008, F-69621, Villeurbanne, France.

Corresponding author, e-mail: frederic.andre@insa-lyon.fr, tel: +33 (0)4 7243 88 16, fax: +33 (0)4 72438811 
Abstract. This paper is devoted to non-uniform approximations for the radiative properties of gases based on effective scaling factors. These methods are recognized as more accurate than other established techniques such as the Curtis-Godson approximation in statistical narrow band modeling, or the Correlated- $k$ assumption. An analytical solution is proposed to calculate these scaling factors and a comprehensive description of the method to derive its parameters from high resolution spectra is given. Practical implications of the results of the present work are quite large, as non-uniform techniques based on effective scaling factors can be applied to any model form. The main value of this work is to render possible and computationally realistic the use of this category of non-uniform approximations for radiative heat transfer calculations.

KEYWORDS: gas radiation, non-uniform, effective scaling factor, $\ell$-distribution, scaled models, Scaled- $k$. 


\section{NOMENCLATURE}

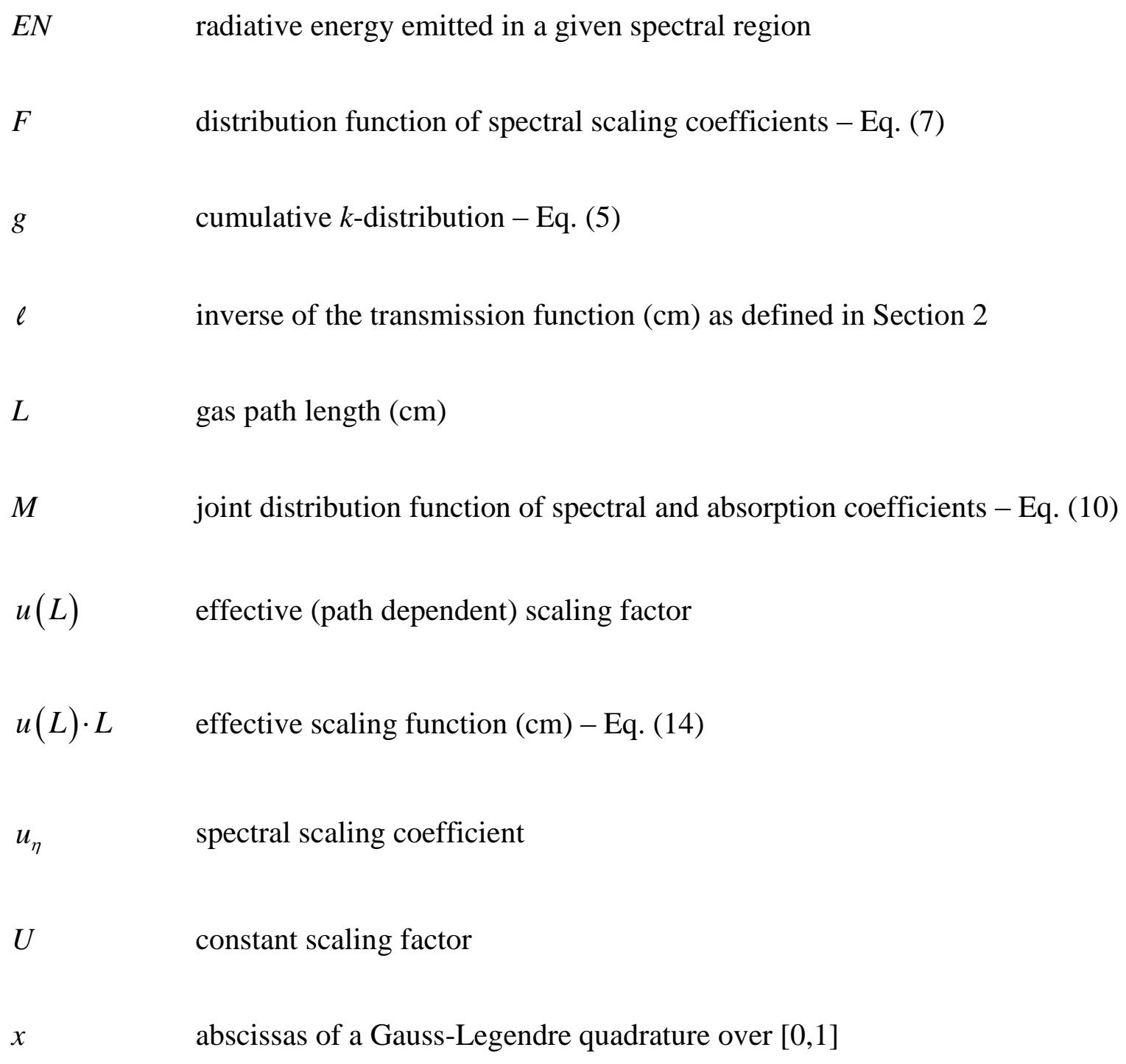

Greek symbols

$\Delta \eta(k) \quad$ set of wavenumbers $\left\{\eta \in \Delta \eta\right.$ such that $\left.k<\kappa_{\eta}^{1}<k+d k\right\}$

$\Delta \eta(k) \quad$ width of the set of wavenumbers $\left\{\eta \in \Delta \eta\right.$ such that $\left.k<\kappa_{\eta}^{1}<k+d k\right\}$

$\kappa_{\eta} \quad$ spectral absorption coefficient $\left(\mathrm{cm}^{-1}\right)$ 


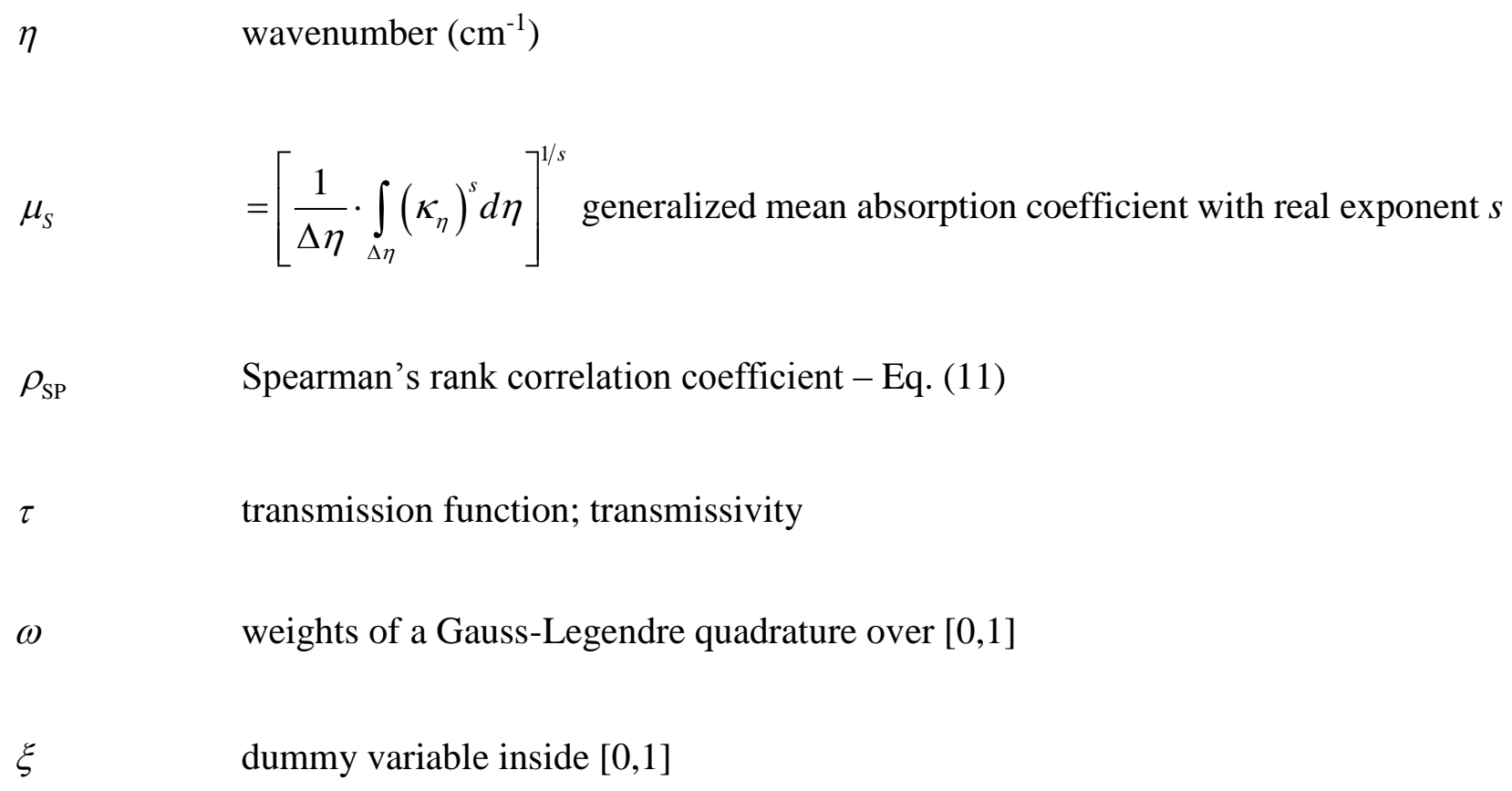

\section{Subscripts}

12 related to the non-uniform path $L=L_{1}+L_{2}$

b blackbody

$P \quad$ Planck mean

$R \quad$ Rosseland mean

S

associated with the set of wavenumbers defined as $\left\{\eta \in \Delta \eta\right.$ such that $\left.\mu_{S}<\kappa_{\eta}^{1}<\mu_{S}+d \mu_{S}\right\}$

\section{Superscripts}

eff effective

GG gray gas 
state 1 or 2 of the gas

$\Delta \eta \quad$ width of the spectral interval for the averaging of spectral properties

\section{Other notations}

$f \circ g$ represents the functional composition of $f$ and $g$ i.e. $f \circ g(x)=f[g(x)]$

$\tau_{i j}^{\Delta \eta}\left(L_{p}, L_{q}\right)=\frac{1}{\Delta \eta} \int_{\Delta \eta} \exp \left(-\kappa_{\eta}^{i} L_{p}-\kappa_{\eta}^{j} L_{q}\right) d \eta \quad E x . \quad \tau_{11}^{\Delta \eta}\left(L_{1}, L_{2}\right)=\frac{1}{\Delta \eta} \int_{\Delta \eta} \exp \left(-\kappa_{\eta}^{1} L_{1}-\kappa_{\eta}^{1} L_{2}\right) d \eta$

$\begin{array}{ll}\begin{array}{ll}\text { Abbreviations } \\ \text { EGA }\end{array} & \text { Emissivity Growth Approximation - Ref. [4] } \\ \text { GWN } & \text { Godson-Weinreb-Neuendorffer's method - Refs. [1,3] } \\ \text { LBL } & \text { Line-By-Line } \\ \text { MoD } & \text { Measure of Dependence - Refs. [12,13] } \\ \text { SNB } & \text { Statistical Narrow Band model - Ref. [1] }\end{array}$




\section{INTRODUCTION}

For many years, the scaling approximation, which consists in assuming that the ratio between two spectra in distinct thermophysical states is a constant with respect to the wavenumbers, was the only possible way to handle radiative heat transfer problems in non-uniform gaseous media. This simple idea, which is known to provide inaccurate results in a general frame as gas spectra in distinct states are not linearly dependent, is however the building block of many non-uniform treatments proposed all along the past century. Various techniques are in fact derived from the scaled view such as:

1/ non-uniform approximations involving two instead of one scaling coefficient, like in the CurtisGodson (CG) approximation [1],

2/ the so-called Correlated- $k$ technique [2], which mostly consists of the definition of scaling coefficients that depend on the values of the absorption coefficient in one particular state chosen as a reference.

3/ the Godson-Weinreb-Neuendorffer GWN [1,3] / Emissivity Growth Approximation EGA [4] or Scaled- $k$ [5] approaches, which do not try to provide an explicit way to evaluate effective scaling factors but instead rely on an implicit definition of these quantities (see Eq. (13) later in this paper).

These last methods are widely recognized as more accurate than those based on explicit definitions of finite numbers of scaling coefficients: see Ref. [1], chapter 12, in which comparisons between the GWN and CG methods are described; or Ref. [5] where it is shown that the Scaled- $k$ approach outperforms the Correlated- $k$ method for radiative heat transfer in highly non-uniform situations. Despite this advantage of non-uniform techniques based on effective scaling factors, it must be recognized that this category of methods has not embraced the same interest as other, in fact usually 
simpler, non-uniform approximations. This is mainly because, in general, solving the implicit equation Eq. (13) to determine the effective scaling factor involves iterative numerical techniques that increase significantly the computational cost of the approach compared to explicit methods.

Recently, the $\ell$-distribution approach was proposed [6]. This approximate model for the radiative properties of gases is founded on a formalism that: 1/ provides accurate approximations in uniform media and $2 /$ allows solving very efficiently the implicit equation involved in the definition of effective scaling factors. While developing this method, the author of the present paper noticed that the literature on effective scaling approximation is quite meager. Indeed, the non-uniform approximation (viz. the implicit equation) is always introduced in an intuitive way without specification of the assumptions that may lead to this particular equation. Accordingly, the existing literature on effective scaling factors does not permit studying in depth some of their properties (the functional form of the solution, for instance) nor it can be helpful to improve the technique further.

The aim of the present paper is to provide insights into the assumptions made to extend the usual concept of constant scaling coefficients to effective ones. Starting from a truly scaled situation, we first explain how the concept of constant scaling coefficient can be extended to real spectra. The main assumption required is shown to be the statistical independence between a spectrum, chosen as a reference, and the spectral scaling coefficients defined as the ratio between any other spectrum and this reference. Based on this assumption of statistical independence, an explicit mathematical formula - Eq. (27) -, which is the core finding of the present work, is derived for effective scaling functions. The full method to construct this function for radiative heat transfer applications is also described and assessed against reference LBL calculations in non-uniform situations. 
The main practical implication of the results provided in the present work is that effective scaling functions require the specification of parameters that are hard to define without optimization. To some extent, one faces the same problem as encountered in Correlated- $k$ models in which the existence of a strictly increasing function that associates spectra in distinct states is assumed. This function cannot be identified directly from LBL data because it is founded on assumptions about the statistical properties of gas spectra, not on a true description of high resolution data. Accordingly, the only way to define this function to associate spectra in distinct states is implicit, by using a relationship that involves the cumulative distributions of the absorption coefficients in the various thermophysical states. In the same way, within the frame of scaled models with variable scaling factors, the most relevant approach to derive effective scaling factors is to solve the implicit equation directly. This makes the e-distribution approach undoubtedly the most efficient and accurate method for this purpose. This statement is discussed further in the paper. The paper also provides an explicit formulation to solve this equation, which can be used with any model form.

The main value of the present paper is to provide, to the best of the author's knowledge, the first detailed analysis of models based on effective scaling factors, from the derivation of the scaling function up to the analysis of the conditions required between spectra for this formulation to be acceptable. Results from the present work have strong implications for future developments of the ८-distribution approach but are not restricted to this approximate model: scaled- $k$ methods, such as FSSK [5] or the recently proposed Scaled-SLW modeling [7], can benefit directly from results described here.

The paper is organized as follows. In the second section, the concept of effective scaling models is introduced. A simple case is treated in order to illustrate some characteristics of this kind of approaches. This section ends with a detailed derivation of effective scaling functions within the 
frame of narrow band models. In the third section, the full method to evaluate the coefficients that appear in the effective scaling function is described. Detailed statistical analysis of spectra together with their correlation with spectral scaling coefficients are given. Comparisons of the approximate model with LBL calculations show the relevancy of the method proposed for radiative heat transfer calculations.

All derivations provided in this work are restricted to the two-cell problem. This analysis is sufficient for application together with all existing methods based on effective scaling factors which either: 1/ assume the existence of some reference state and then scale any other state to this reference [5,7]; or, 2/ propagate the information about scaling factors along a non-uniform path in a step by step manner by coupling adjacent layers $[3,4,6,11]$. In both cases, only two distinct states of the gas are involved. 


\section{MODEL OF EFFECTIVE SCALING FUNCTIONS}

\subsection{Introduction to the concept of effective scaling factors / functions}

Let us start by reminding some results related to scaled spectra. For this purpose, we consider a non-uniform layer discretized in two homogeneous isothermal sub-paths: the first one has a length $L_{1}$ and the gas is in the thermophysical state $\phi_{1}$, the second path has a length $L_{2}$ and the state of the gas is $\phi_{2}$. The spectral absorption coefficients in the two layers are $\kappa_{\eta}^{1}$ and $\kappa_{\eta}^{2}$ respectively. We will restrict here our analysis to narrow bands $\Delta \eta$ over which: 1/ the Planck function is constant; 2/ absorption coefficients $\kappa_{\eta}^{1}$ and $\kappa_{\eta}^{2}$ are strictly positive.

In the case of truly scaled spectra, the ratio $\kappa_{\eta}^{2} / \kappa_{\eta}^{1}=U$ is a constant with respect to the wavenumbers. The transmissivity of the non-uniform path $L=L_{1}+L_{2}$ averaged over the narrow band $\Delta \eta$, written $\tau_{12}^{\Delta \eta}\left(L_{1}, L_{2}\right)$, is:

$$
\tau_{12}^{\Delta \eta}\left(L_{1}, L_{2}\right)=\frac{1}{\Delta \eta} \int_{\Delta \eta} \exp \left(-\kappa_{\eta}^{1} L_{1}-\kappa_{\eta}^{2} L_{2}\right) d \eta=\frac{1}{\Delta \eta} \int_{\Delta \eta} \exp \left[-\kappa_{\eta}^{1}\left(L_{1}+U \cdot L_{2}\right)\right] d \eta
$$

From Eq. (1), we can observe that in the particular case $L_{1}=0$, the following equality holds:

$$
\tau_{12}^{\Delta \eta}\left(L_{1}=0, L_{2}\right)=\tau_{2}^{\Delta \eta}\left(L_{2}\right)=\frac{1}{\Delta \eta} \int_{\Delta \eta} \exp \left(-\kappa_{\eta}^{2} L_{2}\right) d \eta=\frac{1}{\Delta \eta} \int_{\Delta \eta} \exp \left[-\kappa_{\eta}^{1} U \cdot L_{2}\right] d \eta=\tau_{1}^{\Delta \eta}\left(U \cdot L_{2}\right)
$$

It is possible to spread the scaled view from a constant to a non-constant scaling coefficient by using an approach similar to the widely popular $k$-distribution method [2]. Indeed, this technique starts from the concept of gray gas, for which the absorption spectrum $\kappa_{\eta}$ is represented by a single value of the absorption coefficient $k_{P}$ : 


$$
\tau^{\Delta \eta, G G}(L)=\exp \left(-k_{P} \cdot L\right)=\int_{0}^{+\infty} \exp (-k \cdot L) d H\left(k-k_{P}\right)
$$

and extends it to real spectra by a change of variable of integration:

$$
\tau^{\Delta \eta}(L)=\frac{1}{\Delta \eta} \int_{\Delta \eta} \exp \left(-\kappa_{\eta} L\right) d \eta=\int_{0}^{+\infty} \exp (-k \cdot L) d g(k)
$$

where:

$$
g(k)=\mathbb{P}\left(\kappa_{\eta}<k\right)=\frac{1}{\Delta \eta} \cdot \int_{\Delta \eta} H\left(k-\kappa_{\eta}\right) d \eta
$$

is the cumulative $k$-distribution. In Eqs. (3,5), $H$ is the Heaviside step function and notation $\mathbb{P}(Y)$ represents the probability of the "event" $Y$ defined as the fraction of the spectral interval $\Delta \eta$ over which $Y$ is true.

In a similar way, it is possible to extend the scaled model with constant scaling factor:

$$
\tau_{2}^{\Delta \eta}(L)=\tau_{1}^{\Delta \eta}(U \cdot L)=\int_{0}^{+\infty} \tau_{1}^{\Delta \eta}(u \cdot L) d H(u-U)
$$

to real spectra by simply replacing the Heaviside step function by the cumulative distribution of spectral scaling coefficients $u_{\eta}=\kappa_{\eta}^{2} / \kappa_{\eta}^{1}$ defined as:

$$
F(u)=\mathbb{P}\left(u_{\eta}<u\right)=\frac{1}{\Delta \eta} \cdot \int_{\Delta \eta} H\left(u-u_{\eta}\right) d \eta
$$

This yields in the uniform case, i.e. from Eq. (2): 


$$
\tau_{2}^{\Delta \eta}(L)=\int_{0}^{+\infty} \tau_{1}^{\Delta \eta}(u \cdot L) d F(u)
$$

and similarly, for the non-uniform path $L=L_{1}+L_{2}$, viz. starting from Eq. (1):

$$
\tau_{12}^{\Delta \eta}\left(L_{1}, L_{2}\right)=\frac{1}{\Delta \eta} \int_{\Delta \eta} \exp \left(-\kappa_{\eta}^{1} L_{1}-\kappa_{\eta}^{2} L_{2}\right) d \eta=\int_{0}^{+\infty} \tau_{1}^{\Delta \eta}\left(L_{1}+u \cdot L_{2}\right) d F(u)
$$

It is important to notice that the transition from Eq. (6) to Eq. (8) is exact if the gas spectrum $\kappa_{\eta}^{1}$, which then plays the role of a reference state, and the spectral scaling coefficient $u_{\eta}=\kappa_{\eta}^{2} / \kappa_{\eta}^{1}$ are statistically independent. This is because the joint distribution of $\kappa_{\eta}^{1}$ and $u_{\eta}=\kappa_{\eta}^{2} / \kappa_{\eta}^{1}$ defined as:

$$
M(k, u)=\mathbb{P}\left(\kappa_{\eta}^{1}<k, u_{\eta}<u\right)=\frac{1}{\Delta \eta} \cdot \int_{\Delta \eta} H\left(k-\kappa_{\eta}^{1}\right) \cdot H\left(u-u_{\eta}\right) d \eta
$$

is in this particular case equal to the product of distributions $F$, Eq. (7) and $g$, Eq. (5), viz. $M(k, u)=g(k) \cdot F(u)$. This joint distribution is involved in the exact calculation of $\tau_{12}^{\Delta \eta}\left(L_{1}, L_{2}\right)$ in distribution form, as explained in Appendix.

The relevancy of the change of variable involved between Eq. (6) and Eq. (8) is thus strongly connected with the assumption of statistical independence between $\kappa_{\eta}^{1}$ and $u_{\eta}=\kappa_{\eta}^{2} / \kappa_{\eta}^{1}$. This assumption can be assessed by calculating the Spearman's coefficient $\rho_{\mathrm{SP}}$ defined as (see Appendix):

$$
\rho_{\mathrm{SP}}\left(\kappa_{\eta}^{1}, u_{\eta}\right)=12 \cdot \frac{1}{\Delta \eta} \cdot \int_{\Delta \eta} g\left(\kappa_{\eta}^{1}\right) F\left(u_{\eta}\right) d \eta-3
$$


Indeed, as $\rho_{\mathrm{SP}}$ is a so-called Measure of Dependence $\mathrm{MoD}[12,13]$, it takes by definition values between -1 (when the two variables are associated through a strictly decreasing function - variables are then said counter-monotonic) and 1 (when they are related to each other by a strictly increasing function - variables are then said co-monotonic). High absolute values of Spearman's coefficients specify strong relationships (increasing or decreasing) between the variables; small ones are representative of a weak dependence. Null values of Spearman's coefficients indicate the statistical independence between the variables. Calculating Spearman's coefficients thus allows appraising to which extent the assumption of statistical independence between $\kappa_{\eta}^{1}$ and $u_{\eta}=\kappa_{\eta}^{2} / \kappa_{\eta}^{1}$ is correct or not. By extension, it provides information about the possible use of the approximation set by Eq. (9) for the calculation of the transmissivity of the non-uniform path.

The main shortcoming of using Eqs. $(8,9)$ for radiative heat transfer applications is that they require the specification of the distribution function of spectral scaling coefficients which is usually not known. This problem can be circumvented by noticing that the present formulation can be reformulated in implicit form by application of the mean value theorem. This provides a set of equations similar to the one proposed intuitively by Godson in 1953 [8]. Details and comments are given in Appendix. This leads to the following approximation of $\tau_{12}^{\Delta \eta}\left(L_{1}, L_{2}\right)$ :

$$
\tau_{12}^{\Delta \eta}\left(L_{1}, L_{2}\right) \approx \tau_{11}^{\Delta \eta}\left[L_{1}, u\left(L_{2}\right) \cdot L_{2}\right]=\frac{1}{\Delta \eta} \int_{\Delta \eta} \exp \left[-\kappa_{\eta}^{1}\left(L_{1}+u\left(L_{2}\right) \cdot L_{2}\right)\right] d \eta
$$

where the effective scaling factor $u\left(L_{2}\right)$, which depends on the length of the gas path in the column $L_{2}$, is defined as solution of the implicit equation: 


$$
\tau_{2}^{\Delta \eta}\left(L_{2}\right)=\frac{1}{\Delta \eta} \int_{\Delta \eta} \exp \left[-\kappa_{\eta}^{1} u\left(L_{2}\right) \cdot L_{2}\right] d \eta=\tau_{1}^{\Delta \eta}\left[u\left(L_{2}\right) \cdot L_{2}\right]
$$

As the $\ell$-distribution formalism is well suited to non-uniform approximations based on effective scaling factors, it will be used in the subsequent developments. Eq. (13) will thus be written in $\ell-$ distribution form [6] as:

$$
\ell_{1} \circ \tau_{2}^{\Delta \eta}\left(L_{2}\right)=u\left(L_{2}\right) \cdot L_{2}
$$

where $\ell_{1}$ is the inverse of the transmissivity function of the gas in the thermophysical state $\phi_{1}$

defined as $\ell_{1} \circ \tau_{1}^{\Delta \eta}\left(L_{1}\right)=L_{1}$. From now on, the function $\ell_{1} \circ \tau_{2}^{\Delta \eta}\left(L_{2}\right)$ will be called the effective scaling function and $u\left(L_{2}\right)$ the effective scaling factor.

Eqs. (12-14): 1/ mostly extend Eqs. $(1,2)$ to non-scaled spectra through the definition of a path dependent scaling factor, $2 /$ are closely related but not rigorously equivalent to Eqs. $(8,9)$, as explained in Appendix.

Methods based on effective scaling factors thus intuitively and implicitly assume the statistical independence between absorption spectra and spectral scaling coefficients. In practice, this leads to symmetry issues because, in most cases, this assumption is more relevant in one direction, which corresponds to a particular choice of one of the two states as a reference, than in the other one. This property is illustrated in a simple case in the next section.

\subsection{Detailed analysis in a simple case}

The aim of this section is to study a particular situation in order to illustrate some characteristics of approximate models based on effective scaling factors. For this purpose, we consider a non- 
uniform case for which the gas spectrum in state 1 is gray but the second one, in state 2 , can be of any form.

With this couple of states, one has two possible choices for the definition of a reference spectrum and corresponding spectral scaling coefficient: the couple $\kappa_{\eta}^{1}=$ constant $=\kappa_{\eta}^{\text {ref }}$ and $u_{\eta}=\kappa_{\eta}^{2} /$ constant $\left(\right.$ Case S1) or $\kappa_{\eta}^{2}=\kappa_{\eta}^{r e f}$ and $u_{\eta}=\kappa_{\eta}^{1} / \kappa_{\eta}^{2}=$ constant $/ \kappa_{\eta}^{r e f}$ (Case S2).

It is easy to check that application of Eqs. $(12,13)$ in case S1 provides the exact solution (see for instance Appendix B of Ref. [11]). Furthermore, in this case:

- $\quad \kappa_{\eta}^{r e f}$ and $u_{\eta}$ are statistically independent, because the reference spectrum does not depend on wavenumbers whereas the scaling coefficient varies strongly with $\eta$. The Spearman's coefficient for this case is thus equal to 0 ;

- Function $\ell_{1} \circ \tau_{2}^{\Delta \eta}\left(L_{2}\right)=u\left(L_{2}\right) \cdot L_{2}=-\frac{\ln \tau_{2}^{\Delta \eta}\left(L_{2}\right)}{\text { constant }}$ is concave (concave down): this is a consequence of known properties of the so-called Curve-Of-Growth $-\ln \tau_{2}^{\Delta \eta}\left(L_{2}\right)$ [1]. This constraint on function $\ell_{1} \circ \tau_{2}^{\Delta \eta}\left(L_{2}\right)$ is important to warrant the approximation set by Eqs. (12-14) to provide a physically realistic solution. Indeed, the definition of a scaling factor mostly corresponds to an homogenization method where a non-uniform path is "transformed" into an equivalent uniform layer. Guaranteeing $\ell_{1} \circ \tau_{2}^{\Delta \eta}\left(L_{2}\right)$ to be concave (concave down) ensures that this equivalent path actually behaves like a uniform gas layer. This point was raised and studied in Ref. [11].

If we now consider Case S2, we can see that: 
- $u_{\eta}=\kappa_{\eta}^{1} / \kappa_{\eta}^{2}=$ constant $/ \kappa_{\eta}^{r e f}$ is a decreasing function of the reference spectrum. The Spearman's coefficient is in this case -1 ;

- It is difficult to give a priori information about the possible accuracy or physical meaning of the non-uniform approximation.

Accordingly, in this simple case, there exists one obvious choice of reference state (S1) which is more relevant than the other one (S2): it corresponds to the couple $\kappa_{\eta}^{\text {ref }}$ and $u_{\eta}$ that is statistically independent and for which Eqs. $(8,9)$ are exact. We will extend this analysis to the general case in the next section, where an explicit formula for effective scaling functions is also proposed.

\subsection{Mathematical formulation of effective scaling functions}

Let us define $\Delta \eta(k)$ as the width of the set of wavenumbers $\left\{\eta \in \Delta \eta\right.$ such that $\left.k<\kappa_{\eta}^{1}<k+d k\right\}$

where $k$ is a possible value of the spectral absorption coefficient $\kappa_{\eta}^{1}$ and $d k$ is a small positive increment.

The transmissivity of the uniform path $L_{2}$ in the gas in state $\phi_{2}$ averaged over $\Delta \eta(k)$ is:

$$
\tau_{2}^{\Delta \eta(k)}\left(L_{2}\right)=\frac{1}{\Delta \eta(k)} \cdot \int_{\Delta \eta(k)} \exp \left(-\kappa_{\eta}^{2} L_{2}\right) d \eta \approx \frac{1}{\Delta \eta(k)} \cdot \int_{\Delta \eta(k)} \exp \left(-k u_{\eta} L_{2}\right) d \eta
$$

where $u_{\eta}=\kappa_{\eta}^{2} / \kappa_{\eta}^{1}=\kappa_{\eta}^{2} / k$ is the spectral scaling coefficient defined as the ratio between the spectra in the two states. 
As soon as the narrow band $\Delta \eta$ does not contain any transparency region of the gas, the spectral scaling coefficient takes values inside a bounded interval $\left[u_{\min }(k) ; u_{\max }(k)\right]$ and can be written as $u_{\eta}=u_{\min }(k)+u_{\eta}-u_{\min }(k)$. This yields:

$$
\tau_{2}^{\Delta \eta(k)}\left(L_{2}\right)=\exp \left[-k u_{\text {min }}(k) L_{2}\right] \frac{1}{\Delta \eta(k)} \cdot \int_{\Delta \eta(k)} \exp \left(-k\left[u_{\eta}-u_{\text {min }}(k)\right] L_{2}\right) d \eta
$$

One can write the integral at the RHS in Eq. (16) as:

$$
\frac{1}{\Delta \eta(k)} \cdot \int_{\Delta \eta(k)} \exp \left(-k\left[u_{\eta}-u_{\min }(k)\right] L_{2}\right) d \eta=\exp \left[-k \cdot R\left(k, L_{2}\right)\right]
$$

in which we have introduced the notation:

$$
R\left(k, L_{2}\right)=-\frac{1}{k} \ln \left[\frac{1}{\Delta \eta(k)} \cdot \int_{\Delta \eta(k)} \exp \left(-k\left[u_{\eta}-u_{\min }(k)\right] L_{2}\right) d \eta\right]
$$

Function $R\left(k, L_{2}\right)$ can be equivalently written as:

$$
R\left(k, L_{2}\right)=-\frac{1}{k} \ln \left(1-\frac{1}{\Delta \eta(k)} \cdot \int_{\Delta \eta(k)}\left[1-\exp \left(-k\left[u_{\eta}-u_{\min }(k)\right] L_{2}\right)\right] d \eta\right)
$$

Let us write $I_{b, \eta}\left(T_{2}\right)$ the Planck function at temperature $T_{2}$ assumed to be constant over the narrow band $\Delta \eta$. The quantity $I_{b, \eta}\left(T_{2}\right) \cdot \int_{\Delta \eta(k)}\left[1-\exp \left(-k\left[u_{\eta}-u_{\min }(k)\right] L_{2}\right)\right] d \eta$ represents the amount of radiative energy $E N$ emitted in the spectral region defined as $\left\{\eta \in \Delta \eta\right.$ such that $k<\kappa_{\eta}^{1}<k+d k$ and $\left.u_{\eta}-u_{\text {min }}(k)>0\right\}$. This quantity can be reasonably 
considered small compared to the energy $E N_{b}$ emitted by a blackbody over the same spectral interval which is $E N_{b}=I_{b, \eta}\left(T_{2}\right) \cdot \Delta \eta(k)$. Accordingly, Eq. (19) can be approximated as:

$$
R\left(k, L_{2}\right)=-\frac{1}{k} \ln \left(1-\frac{E N}{E N_{b}}\right) \approx \frac{E N}{k \cdot E N_{b}}=\frac{1}{k} \frac{1}{\Delta \eta(k)} \cdot \int_{\Delta \eta(k)}\left[1-\exp \left(-k\left[u_{\eta}-u_{\min }(k)\right] L_{2}\right)\right] d \eta
$$

This provides the following formula for the effective scaling function over the interval $\Delta \eta(k)$ :

$$
u_{\min }(k) L_{2}+R\left(k, L_{2}\right) \approx u_{\min }(k) L_{2}+\frac{1}{k} \frac{1}{\Delta \eta(k)} \cdot \int_{\Delta \eta(k)}\left[1-\exp \left(-k\left[u_{\eta}-u_{\min }(k)\right] L_{2}\right)\right] d \eta
$$

Let us now consider the optically thin limit for which $L_{2} \rightarrow 0$. In this case, the following estimate of Eq. (21) can be used as an approximation of the effective scaling function over $\Delta \eta(k)$ :

$$
u_{\min }(k) L_{2}+\frac{1}{k} \frac{1}{\Delta \eta(k)} \cdot \int_{\Delta \eta(k)}\left[1-\exp \left(-k\left[u_{\eta}-u_{\min }(k)\right] L_{2}\right)\right] d \eta \approx \frac{1}{\Delta \eta(k)} \cdot \int_{\Delta \eta(k)} u_{\eta} d \eta \cdot L_{2}
$$

The integral at the RHS in Eq. (22) is the mean value of spectral scaling coefficients over $\Delta \eta(k)$. If this value is the same for all $k$, then mean absorption coefficients inside distinct intervals $\Delta \eta(k)$ and $\Delta \eta\left(k^{\prime}\right), k^{\prime} \neq k$ are translated by the same amount between the two states: accordingly, gas spectra averaged over $\Delta \eta(k)$ can be treated as scaled since a unique scaling factor can be used for all these intervals. 
A similar analysis can be made at the optically thick limit for which:

$$
u_{\text {min }}(k) L_{2}+\frac{1}{k} \frac{1}{\Delta \eta(k)} \cdot \int_{\Delta \eta(k)}\left[1-\exp \left(-k\left[u_{\eta}-u_{\min }(k)\right] L_{2}\right)\right] d \eta \approx u_{\min }(k) L_{2}
$$

Gas spectra can be treated as rigorously scaled once averaged over $\Delta \eta(k)$ for large gas path lengths as soon as $u_{\min }(k)$ is the same for all $k$.

This shows that if the reference absorption spectrum and the spectral scaling coefficients are statistically independent, then the functional form set by Eq. (21), which depends on $k$, can be represented, at the optically thin and thick limits, by a unique function that does not depend on the values taken by the absorption coefficient.

This property can be extended to any gas path length. However, as functions $\underbrace{u_{\text {min }}(k)}_{=u_{\min }} L_{2}+R\left(k, L_{2}\right)$ are dependent on $k$-values through the non-linear dependence of $R\left(k, L_{2}\right)$ with $k$, an additional assumption is required. In order to extend the functional form Eq. (21) to the entire narrow band and only keep its dependence with respect to the length of the gas path $L_{2}$, we will assume here for simplicity that one can define a mean value of $k$, written from now on $\mu_{s}$, such that for any length:

$$
\tau_{2}^{\Delta \eta}\left(L_{2}\right)=\tau_{1}^{\Delta \eta}\left[u_{\min }\left(\mu_{S}\right) L_{2}+R\left(\mu_{S}, L_{2}\right)\right]
$$

Notice that a possible dependence of $u_{\min }$ and $R$ with $\mu_{S}$ is retained explicit in this formulation because in practice gas spectra and scaling coefficients are never rigorously independent: Spearman's coefficients are not null when real spectra are considered. Letting these quantities as 
non-constant will allow us to search for their best values through an optimization process, as will be discussed in the next section.

The derivation provided in this section can be applied to any model form. However, we will use in the subsequent developments the -distribution formalism [6] which is particularly well suited to non-uniform approximations based on effective scaling functions. Accordingly, Eq. (21) is rewritten:

$$
\begin{aligned}
\ell_{1} \circ \tau_{2}^{\Delta \eta}\left(L_{2}\right) & =u_{\text {min }}\left(\mu_{S}\right) L_{2}+\frac{1}{\mu_{S}} \frac{1}{\Delta \eta\left(\mu_{S}\right)} \cdot \int_{\Delta \eta\left(\mu_{S}\right)}\left[1-\exp \left(-\mu_{S}\left[u_{\eta}-u_{\text {min }}\left(\mu_{S}\right)\right] L_{2}\right)\right] d \eta \\
& =u_{\text {min }}\left(\mu_{S}\right) L_{2}+\frac{1}{\mu_{S}} \int_{0}^{+\infty}\left[1-\exp \left(-\mu_{S}\left[u-u_{\text {min }}\left(\mu_{S}\right)\right] L_{2}\right)\right] d F_{S}(u)
\end{aligned}
$$

where $F_{S}$ is the distribution function of spectral scaling coefficients restricted to the spectral interval $\left\{\eta \in \Delta \eta\right.$ such that $\left.\mu_{S}<\kappa_{\eta}^{1}<\mu_{S}+d \mu_{S}\right\}$. It may be close but somewhat different from the distribution of scaling coefficients over the narrow band in cases of weak dependence i.e. for nonzero values of Spearman's coefficients. $F_{S}$ is defined as:

$$
F_{S}(u)=\mathbb{P}\left(u_{\eta}<u \text { and } \kappa_{\eta} \approx \mu_{S}\right)=\frac{1}{\Delta \eta\left(\mu_{S}\right)} \cdot \int_{\Delta \eta\left(\mu_{S}\right)} H\left(u-u_{\eta}\right) d \eta
$$

For practical use (see the next section), we will rewrite the effective scaling function Eq. (25) under the following form:

$$
\ell_{1} \circ \tau_{2}^{\Delta \eta}\left(L_{2}\right)=u_{\text {min }}\left(\mu_{S}\right) L_{2}+\frac{1}{\mu_{S}} \int_{0}^{1}\left[1-\exp \left(-\mu_{S}\left[F_{S}^{-1}(\xi)-u_{\text {min }}\left(\mu_{S}\right)\right] L_{2}\right)\right] d \xi
$$


Transition from Eq. (25) to Eq. (27) is done by application of the change of variable $F_{S}(u) \leftarrow \xi \in[0,1] \Leftrightarrow u=F_{S}^{-1}(\xi)$. It can be noticed that by definition of $F_{S}$, one has $F_{S}^{-1}(\xi) \geq u_{\min }\left(\mu_{S}\right)$ for any $\xi \in[0,1]$ and thus the integral in Eq. (27) is positive.

The relevance of the functional form Eq. (27) (called a Bernstein function in Mathematical Sciences [9] or a Laplace exponent of a Levy subordinator in Probabilistic literature [10]) to represent $\ell_{1} \circ \tau_{2}^{\Delta \eta}\left(L_{2}\right)$ will be studied further in Section 3 .

One can notice that:

1/ Eq. (27) provides the solution to Eq. (13) if the following representation for the effective scaling factor is used:

$$
u\left(L_{2}\right)=\frac{\ell_{1} \circ \tau_{2}^{\Delta \eta}\left(L_{2}\right)}{L_{2}}=u_{\min }\left(\mu_{S}\right)+\frac{1}{L_{2} \cdot \mu_{S}} \int_{0}^{1}\left[1-\exp \left(-\mu_{S}\left[F_{S}^{-1}(\xi)-u_{\min }\left(\mu_{S}\right)\right] L_{2}\right)\right] d \xi(28)
$$

$2 /$ if $\ell_{1} \circ \tau_{2}^{\Delta \eta}(L)$ can be written as in Eq. (27), then $\ell_{2} \circ \tau_{1}^{\Delta \eta}(L)$, which is its inverse, cannot share the same mathematical representation if gas spectra are not truly scaled, i.e. if the integral at the RHS in Eq. (28) is not null. This is because if an increasing function is concave (concave down), then its inverse is convex (concave up) which allows writing [11]:

$$
\begin{aligned}
\frac{\partial^{2} \ell_{1} \circ \tau_{2}^{\Delta \eta}(L)}{\partial L^{2}} & =-\mu_{S} \int_{0}^{1}\left[F_{S}^{-1}(\xi)-u_{\min }\left(\mu_{S}\right)\right]^{2} \exp \left(-\mu_{S}\left[F_{S}^{-1}(\xi)-u_{\min }\left(\mu_{S}\right)\right] L_{2}\right) d \xi \leq 0 \\
& \Rightarrow \frac{\partial^{2} \ell_{2} \circ \tau_{1}^{\Delta \eta}(L)}{\partial L^{2}} \geq 0
\end{aligned}
$$


The second inequality is not compatible with a possible representation of $\ell_{2} \circ \tau_{1}^{\Delta \eta}(L)$ under the same form as in Eq. (27), by definition of Bernstein functions, viz. a function $f$ is called Bernstein if it takes positive values and $(-1)^{n-1} \partial^{n} f / \partial x^{n} \geq 0$.

3/ this general formula complies with the mathematical formulation of $\ell_{1} \circ \tau_{2}^{\Delta \eta}(L)$ provided in Ref. [11] which was restricted to the SNB model for Lorentz lines with Malkmus' distribution of linestrengths or the $\ell$-distribution approach with close thermophysical states $\left(\phi_{1}, \phi_{2}\right)$. More details can be found in Ref. [11]. 


\section{APPLICATION}

In the following section, details about the method proposed to construct an effective scaling function are given. This function is then applied for radiative heat transfer calculations and assessed against reference non-uniform LBL calculations and exact solutions of the implicit equation Eq. (13). Comparisons with outputs of the $\ell$-distribution approach are also provided.

\subsection{Model parameters and mathematical tools}

The high resolution $\left(10^{-2} \mathrm{~cm}^{-1}\right)$ LBL dataset used for the reference calculations was described previously in Ref. [14]. It is based on HITEMP2010 [15] for $\mathrm{H}_{2} \mathrm{O}$, which is the only radiating species considered here. In order to assess the full methodology to define effective scaling functions, a uniform model needs to be selected. Here, the LBL approach was chosen. The Fortran subroutine SUBSPEAR taken from Ref. [16] was implemented to evaluate Spearman's coefficients. It is based on a method that differs from a direct application of Eq. (11) but was found to provide the same results at a lower computational cost. The code to generate shifted GaussLegendre quadratures (see Section 3.2.2.) was taken from the same reference. The fitting process (see Sections 3.2.3 and 3.2.4) is handled by a Stochastic Particle Swarm Optimizer (SPSO). This method was chosen for convenience: any other optimization technique (Levenberg-Marquardt, etc.) can be used for this purpose.

The -distribution model parameters, used in the present work for comparison with the method based on explicit effective scaling factors, viz. Eq. (27), are the same as those described in Ref. [6]. The interested readers should refer to this paper for additional details. 


\subsection{Detailed method to estimate the effective scaling function}

The method to estimate effective scaling functions requires several steps. They are described in this section.

\subsubsection{Statistical test of independence between gas spectra and spectral scaling coefficients}

For any pair of absorption spectra $\left(\kappa_{\eta}^{1}, \kappa_{\eta}^{2}\right)$, it is possible to construct two couples of variables consisting of a "reference" absorption spectrum (which plays the role of $\kappa_{\eta}^{1}$ in Section 2) and a corresponding spectral scaling coefficient (constructed by dividing the other spectrum by this "reference"). These two couples are $\left(\kappa_{\eta}^{1}, \kappa_{\eta}^{2} / \kappa_{\eta}^{1}\right)$ and $\left(\kappa_{\eta}^{2}, \kappa_{\eta}^{1} / \kappa_{\eta}^{2}\right)$. For each of them, one can evaluate over any narrow band a value of Spearman's coefficient, $\rho_{\mathrm{SP}}\left(\kappa_{\eta}^{1}, \kappa_{\eta}^{2} / \kappa_{\eta}^{1}\right)$ and $\rho_{\mathrm{SP}}\left(\kappa_{\eta}^{2}, \kappa_{\eta}^{1} / \kappa_{\eta}^{2}\right)$. These coefficients can be plotted as a function of the narrow band centers. An output of such a calculation is depicted in Figure 1, for a couple of states at the gas with the same molar fractions of $\mathrm{H}_{2} \mathrm{O}(0.2)$ and $\mathrm{N}_{2}(0.8)$, the same total pressure (1 atm) but distinct temperatures (1000 K and $2000 \mathrm{~K})$.

Clearly, there are in most cases large differences between the two values of Spearman's coefficients: one of them remains relatively small (indicating weak dependence between the spectrum chosen as the reference and the corresponding scaling coefficient); the second one has large negative values indicating a strong decreasing relationship between the variables. This means that the assumption of statistical independence is more reasonable in one direction (which corresponds to the couple of reference absorption coefficient and scaling coefficient associated with the smallest absolute value of $\rho_{\mathrm{SP}}$ ) than in the other one. 


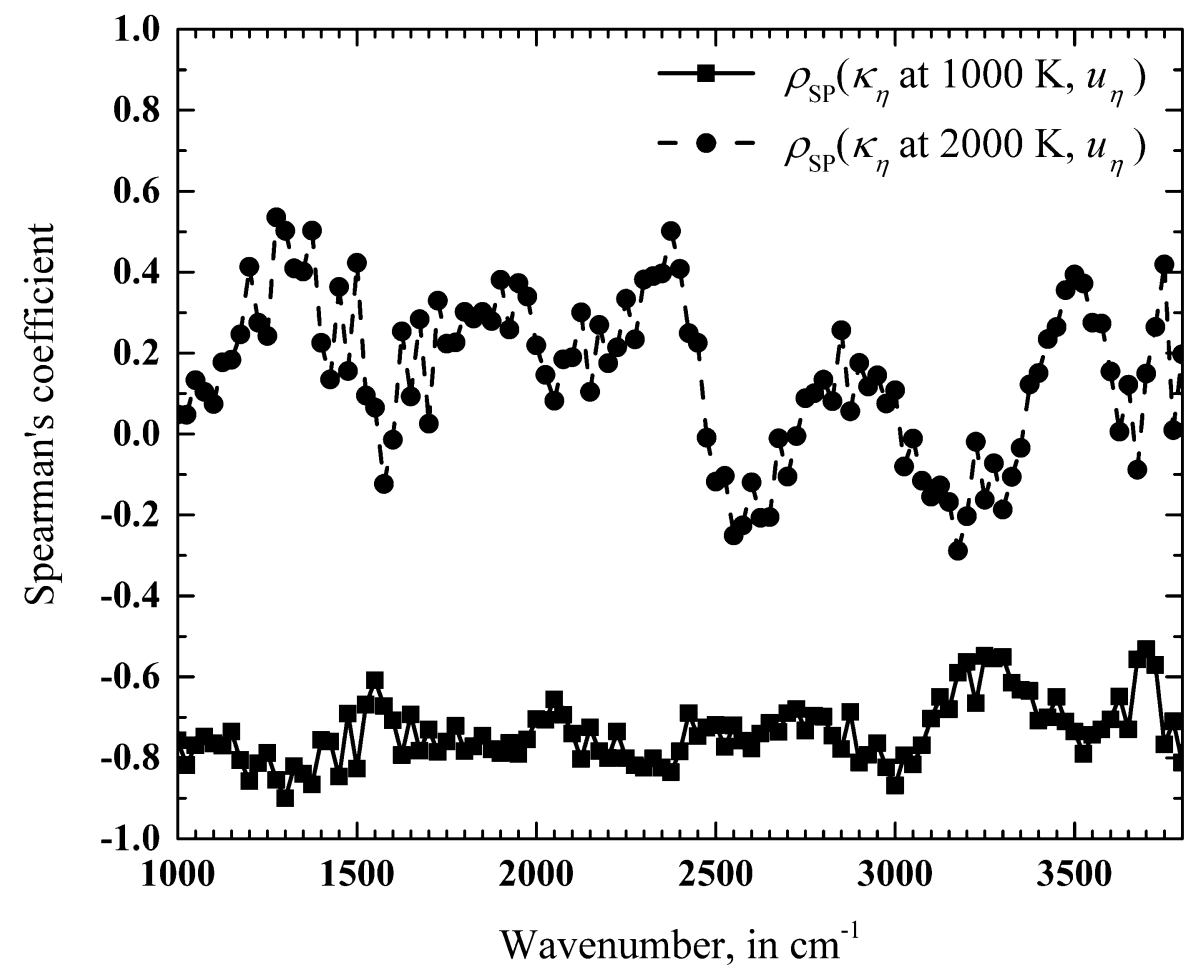

Figure 1. Asymmetry in Spearman's coefficients - Case C3

This is confirmed by the plots provided in Figures 2 that depict the reordered absorption and spectral scaling coefficients reordered with the same rearrangement of the wavenumber scale as for the absorption spectrum. Results correspond to the narrow band centered at $1700 \mathrm{~cm}^{-1}$. In order to avoid strong oscillations of the spectral scaling coefficients, a 64 points Savitsky-Golay smoothing filter was applied to the data. Figure 2-A (the reference spectrum is the hot one) shows a very weak dependence between the spectrum and the scaling coefficient. Corresponding value of Spearman's $\rho_{\mathrm{SP}}$ is equal to 0.02 . In the other direction (the reference spectrum is the cold one), see Figure 2-B, the scaling coefficient appears to be a decreasing function of the reference absorption coefficient. Spearman's $\rho_{\mathrm{SP}}$ for this case is -0.73 . Notice also that assuming the reference spectrum and scaling coefficient to be independent in the case of Figure 2-B would consist in replacing the decreasing curve by some mean line as in Figure 2-A. This may increase artificially the highest 
values of the absorption coefficient observed near $\xi=1$ and produce a physically unrealistic behavior at the optically thin limit. A symmetrical effect may be also observed at the optically thick limit.

Accordingly, the calculation of Spearman's $\rho_{\mathrm{SP}}$ allows choosing the most appropriate couples $\left(\kappa_{\eta}^{1}, \kappa_{\eta}^{2} / \kappa_{\eta}^{1}\right)$ or $\left(\kappa_{\eta}^{2}, \kappa_{\eta}^{1} / \kappa_{\eta}^{2}\right)$ to seek an effective scaling function under the mathematical form Eq. (27). This is because the derivation of this formula is strongly connected with the assumption of statistical independence between the reference spectrum and the corresponding spectral scaling coefficients.

We have thus used the following selection rule:

$$
\left|\rho_{\mathrm{SP}}\left(\kappa_{\eta}^{1}, \kappa_{\eta}^{2} / \kappa_{\eta}^{1}\right)\right| \leq\left|\rho_{\mathrm{SP}}\left(\kappa_{\eta}^{2}, \kappa_{\eta}^{1} / \kappa_{\eta}^{2}\right)\right| \Rightarrow\left\{\begin{array}{l}
\kappa_{\eta}^{1} \text { is the reference spectrum } \\
\text { and } \kappa_{\eta}^{2} / \kappa_{\eta}^{1} \text { the corresponding scaling coefficien }
\end{array}\right.
$$

in which case all equations provided in Section 2 can be used directly, or:

$$
\left|\rho_{\mathrm{SP}}\left(\kappa_{\eta}^{2}, \kappa_{\eta}^{1} / \kappa_{\eta}^{2}\right)\right| \leq\left|\rho_{\mathrm{SP}}\left(\kappa_{\eta}^{1}, \kappa_{\eta}^{2} / \kappa_{\eta}^{1}\right)\right| \Rightarrow\left\{\begin{array}{l}
\kappa_{\eta}^{2} \text { is the reference spectrum } \\
\text { and } \kappa_{\eta}^{1} / \kappa_{\eta}^{2} \text { the corresponding scaling coefficient }
\end{array}\right.
$$

in which case all equations in Section 2 can also be used but the roles played by indices and exponents " 1 " and " 2 " need to be switched. The necessity to define selection rules $(30,31)$ is the main reason that explains the asymmetries observed in non-uniform approximations based on effective scaling factors $[1,3,8,11]$.

For all the cases considered in this work (details are provided in Section 3.3), the most appropriate direction of calculation was always found to correspond to the choice of the hot gas as the reference 
(except for some narrow bands). Comparisons with the criterion proposed in Ref. [11], based on ratios of Planck and Rosseland mean absorption coefficients, revealed that these two criteria (Spearman's coefficient and ratio of mean absorption coefficients) provide the same outcome for most narrow bands. Details are provided in Section 3.3. 


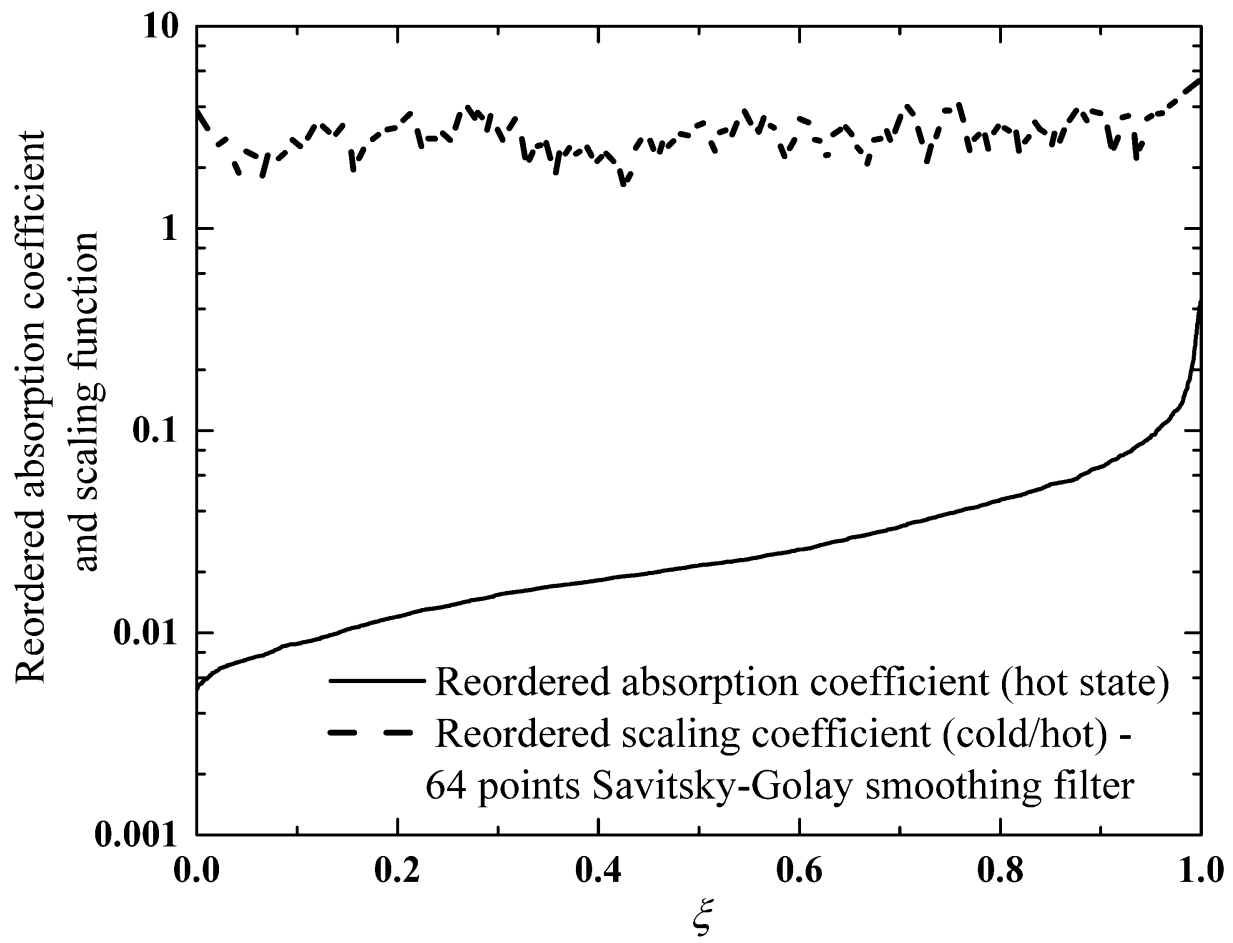

Figure 2-A. Reordered absorption and scaling coefficients - Reference is the hot gas.

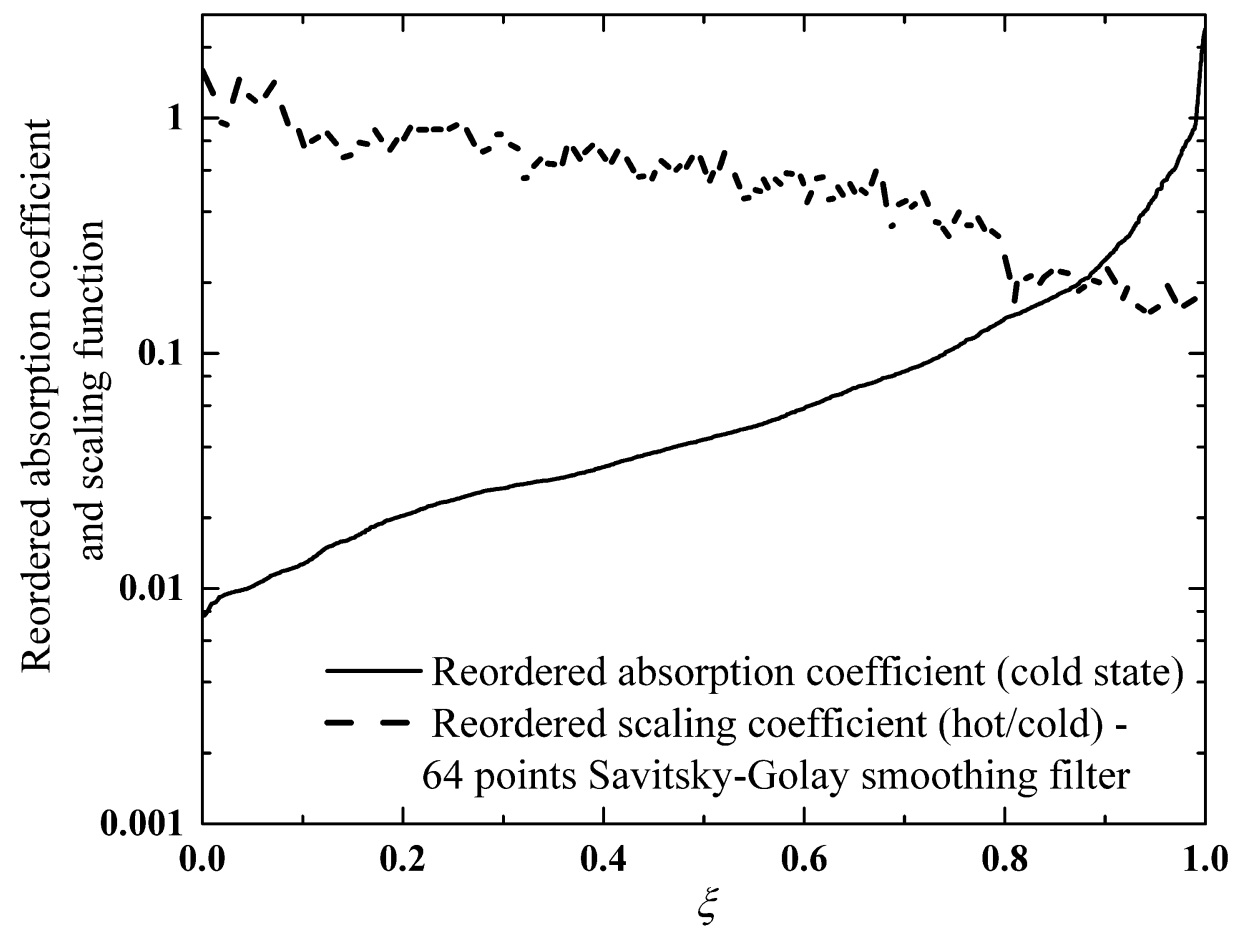

Figure 2-B. Reordered absorption and scaling coefficients - Reference is the cold gas. 


\subsubsection{Preliminary calculations}

As soon as the direction for the model is chosen according to the criteria Eqs. (30,31) (state " 1 " is assumed to be the reference state all along this section), the second step of the method consists in finding an approximate value for the generalized mean $\mu_{S}$. This is done by assuming that the reference absorption coefficient and the corresponding spectral scaling coefficient are actually statistically independent over the narrow band. In this case, $F_{S}$ (Eq. (26)) can be replaced by $F$ (Eq. (7)). The quantity $u_{\text {min }}\left(\mu_{S}\right)$ can also be set to the minimum value of spectral scaling coefficient found over the narrow band. With these assumptions, Eq. (27) becomes:

$$
\ell_{1} \circ \tau_{2}^{\Delta \eta}\left(L_{2}\right)=u_{\text {min }} L_{2}+\frac{1}{\mu_{S}} \int_{0}^{1}\left[1-\exp \left(-\mu_{S}\left[F^{-1}(\xi)-u_{\text {min }}\right] L_{2}\right)\right] d \xi
$$

Then, the integral in Eq. (32) is approximated as a weighted sum:

$$
\ell_{1} \circ \tau_{2}^{\Delta \eta}\left(L_{2}\right) \approx u_{\min } L_{2}+\frac{1}{\mu_{S}} \sum_{i=1}^{N} \omega_{i}\left[1-\exp \left(-\mu_{S}\left[F^{-1}\left(x_{i}\right)-u_{\min }\right] L_{2}\right)\right]
$$

where $\omega_{i}, x_{i}, i=1, N$ are the weights and abscissas of a Gauss-Legendre quadrature at order $N$ (=6 in the present work) over the $[0,1]$ interval. The method to construct such an approximation of the integral is similar to the technique widely encountered in $k$-distribution methods, but applied here to the distribution function of scaling coefficients instead of the $k$-distribution.

Then, the approximation set by Eq. (33) is used in a non-linear least square process so as to minimize the following objective function $\operatorname{Dist}\left(\mu_{S}\right)$ (the optimization process thus consists in finding $\mu_{S}^{0}$ such that $\left.\operatorname{dDist}\left(\mu_{S}=\mu_{S}^{0}\right) / d \mu_{S}=0\right)$ : 


$$
\left\{\begin{array}{l}
\operatorname{Dist}\left(\mu_{S}\right)=\sum_{j=1}^{J}\left[\tau_{2}^{\Delta \eta}\left(L_{j}\right)-\tau_{1}^{\Delta \eta}\left(L_{j}^{\text {eff }}\right)\right]^{2} \\
L_{j}^{e f f}=u_{\min } L_{j}+\frac{1}{\mu_{S}} \sum_{i=1}^{N} \omega_{i}\left[1-\exp \left(-\mu_{S}\left[F^{-1}\left(x_{i}\right)-u_{\min }\right] L_{j}\right)\right]
\end{array}\right.
$$

In Eq. (34), $L_{j}^{\text {eff }}, j=1, J$ are equivalent gas path lengths. They correspond to the values of the effective scaling function at lengths $L_{j}, j=1, J$. These equivalent gas path lengths only depend on $\mu_{S}$.

Lengths $L_{j}, j=1, J$ are chosen logarithmically scaled inside an interval $\left[L_{\min }, L_{\max }\right]$ whose boundaries are defined as solutions of:

$$
\tau_{2}^{\Delta \eta}\left(L_{\min }\right)=0.99, \tau_{2}^{\Delta \eta}\left(L_{\max }\right)=0.01
$$

Eqs. (35) are solved using a bisection method. $J$ was arbitrarily set to 20 .

At this step, we thus have approximations for $u_{\text {min }}\left(\mu_{S}\right) \approx u_{\text {min }}, \mu_{S} \approx \mu_{S}^{0}$ (where $\mu_{S}^{0}$ is the solution of the optimization process set by Eq. (34)) as well as the distribution function of scaling coefficients over the arrow band $\Delta \eta, F$ (as defined by Eq. (7)) and associated values of $F^{-1}\left(x_{i}\right), i=1, N$.

\subsubsection{Treatment of the integral at the RHS in Eq. (27)}

If $\mu_{S}$ is known, it is theoretically possible to estimate $F_{S}(u)$ following its definition i.e. Eq. (26). However, in practice, using this definition is not convenient as it requires evaluating the conditional probability of scaling coefficients given the constraint $\kappa_{\eta}=\mu_{S}$. Accordingly, we propose to circumvent this difficulty by reformulating Eq. (27) into: 


$$
\begin{aligned}
\ell_{1} \circ \tau_{2}^{\Delta \eta}\left(L_{2}\right) & =u_{\text {min }}\left(\mu_{S}\right) L_{2}+\frac{1}{\mu_{S}} \int_{0}^{1}\left[1-\exp \left(-\mu_{S}\left[\frac{F_{S}^{-1}(\xi)}{F^{-1}(\xi)} F^{-1}(\xi)-u_{\min }\left(\mu_{S}\right)\right] L_{2}\right)\right] d \xi \\
& =u_{\text {min }}\left(\mu_{S}\right) L_{2}+\frac{1}{\mu_{S}} \int_{0}^{1}\left[1-\exp \left(-\mu_{S}\left[D(\xi) F^{-1}(\xi)-u_{\min }\left(\mu_{S}\right)\right] L_{2}\right)\right] d \xi
\end{aligned}
$$

where:

$$
D(\xi)=\frac{F_{S}^{-1}(\xi)}{F^{-1}(\xi)}
$$

is a weighting factor. Its role is to rectify the moments of the scaling coefficients defined over the narrow band $\Delta \eta$ so as to coincide with those over the unknown interval $\Delta \eta\left(\mu_{S}\right)$. These differences between the moments are due to the fact that $\kappa_{\eta}$ and $u_{\eta}$ are not rigorously independent (see Figure 1, for instance) and thus $F_{S} \neq F$. Accordingly, the moments of $u_{\eta}$ over $\Delta \eta\left(\mu_{S}\right)$, viz. $\int_{0}^{1}\left[F_{S}^{-1}(\xi)\right]^{n} d \xi, n \in \mathbb{N}$, and over $\Delta \eta$, i.e. $\int_{0}^{1}\left[F^{-1}(\xi)\right]^{n} d \xi, n \in \mathbb{N}$, are not exactly the same. If $\kappa_{\eta}$ and $u_{\eta}$ are statistically independent over $\Delta \eta, F_{S}=F$ and, following Eq. (37), $D(\xi)=1$.

Eq. (36) can be written in the same form as Eq. (33):

$$
\ell_{1} \circ \tau_{2}^{\Delta \eta}\left(L_{2}\right) \approx u_{\text {min }}\left(\mu_{S}\right) L_{2}+\frac{1}{\mu_{S}} \sum_{i=1}^{N} \omega_{i}\left[1-\exp \left(-\mu_{S}\left[D\left(x_{i}\right) \cdot F^{-1}\left(x_{i}\right)-u_{\text {min }}\left(\mu_{S}\right)\right] L_{2}\right)\right]
$$

The coefficients that appear in Eq. (38), viz. $u_{\text {min }}\left(\mu_{S}\right), \mu_{S}$ and $D\left(x_{i}\right), i=1, N$ can be found by a non-linear least square fitting technique. This is detailed in the next section. 


\subsubsection{Non-linear least square optimization}

The final step consists in a non-linear least square fitting of the "true" parameters of the model. Initial values and bounds of the search space are provided in Table 1 . They are defined in terms of the approximations found in Section 3.2.2.

\begin{tabular}{|c|c|c|c|}
\hline Parameter & Initial value & Lower bound & Upper bound \\
\hline$u_{\min }\left(\mu_{S}\right)$ & $u_{\min }$ & $0.1 u_{\min }$ & $10 u_{\min }$ \\
\hline$\mu_{S}$ & $\mu_{S}^{0}$ & $0.1 \mu_{S}^{0}$ & $10 \mu_{S}^{0}$ \\
\hline$D\left(x_{i}\right), i=1, N$ & 1 & 0.1 & 10 \\
\hline
\end{tabular}

Table 1. Initial parameter values and bounds of the search space used in the optimization process.

A SPSO was used to minimize the following objective function, similar to Eq. (34) but that now depends on more than one variable:

$$
\left\{\begin{array}{l}
\operatorname{Dist}\left[\mu_{S} ; u_{\min }\left(\mu_{S}\right) ; D\left(x_{i}\right), i=1, N\right]=\sum_{j=1}^{J}\left[\tau_{2}^{\Delta \eta}\left(L_{j}\right)-\tau_{1}^{\Delta \eta}\left(L_{j}^{e f f}\right)\right]^{2} \\
L_{j}^{e f f}=u_{\min }\left(\mu_{S}\right) L_{j}+\frac{1}{\mu_{S}} \sum_{i=1}^{N} \omega_{i}\left[1-\exp \left(-\mu_{S}\left[D\left(x_{i}\right) F^{-1}\left(x_{i}\right)-u_{\min }\left(\mu_{S}\right)\right] L_{j}\right)\right]
\end{array}\right.
$$

At the end of the optimization process, we thus have estimates for all the quantities that appear in the model of effective scaling function. These parameters can be stored in tables for later use (which means that the full method does not need to be applied for each new calculation but a database can be constructed using the present approach to build a scaled model dataset). 
An example of output of the fitting process is illustrated in Figure 3-A. It corresponds to the Case $\mathrm{C} 2$ described in the next section, over the narrow band centered at $1700 \mathrm{~cm}^{-1}$. The LBL curve corresponds to the exact transmissivity function at temperature $1000 \mathrm{~K}$; the squares depict the transmission obtained by application of a LBL model at the hot temperature (here $1500 \mathrm{~K}$ ) with the effective scaling factors obtained by the method described in this section. The corresponding effective scaling function $\ell_{1} \circ \tau_{2}^{\Delta \eta}\left(L_{2}\right)$ is compared to the exact values of solutions of Eq. (13) obtained by a bisection method in Figure 3-B.

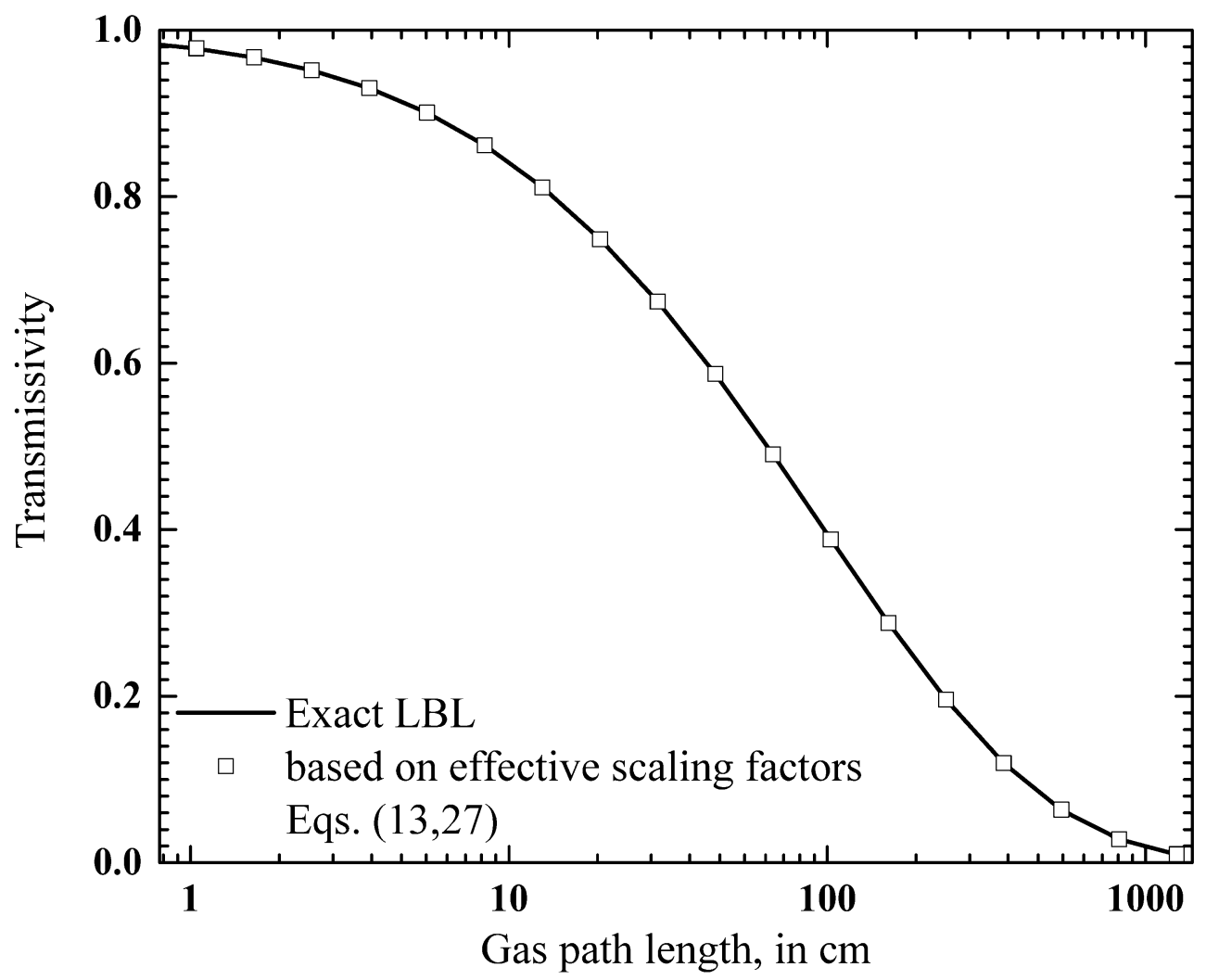

Figure 3-A. Uniform transmissivities (see text for details) 


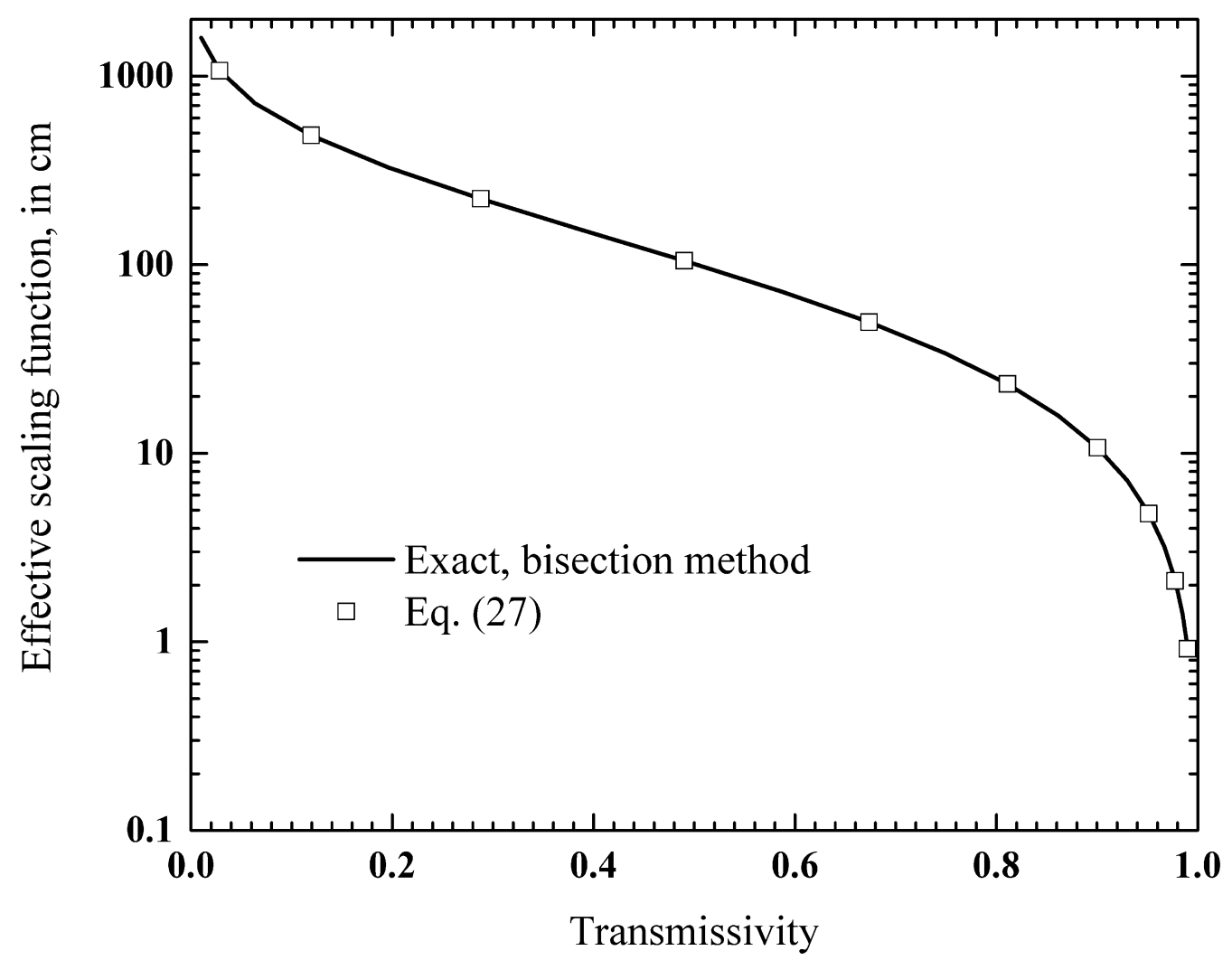

Figure 3-B. Effective scaling function (see text for details)

\subsection{Application of effective scaling factors for radiative heat transfer calculations}

For all the test cases considered in this section, the non-uniform LBL calculation is the reference. The following terminology is used to distinguish the various approximate models:

- "Exact $\tau_{11}$ " corresponds to the use of Eq. (12) with the scaling factor $u\left(L_{2}\right)$ obtained by solving Eq. (13) with the bisection method;

- "Exact $\tau_{22}$ " is the same as "Exact $\tau_{11}$ " but the roles played by states 1 and 2 are reversed;

- " $\tau_{i i}$ with effective scaling function" is the same as "Exact $\tau_{11}$ " or "Exact $\tau_{22}$ " with the scaling function calculated as described in Section 3.2. - the scaling function and the value of index $i$ is determined over each narrow band by application of the criteria $(30,31)$; 
- $\quad$-distribution is the output of the model described in Ref. [6] - for this model, the ordering of the path uses the criterion set in Ref. [11] (which, as noticed earlier in the present paper, coincides with $(30,31)$ in most cases).

Index " 2 " is always associated with the cold layer, index " 1 " with the hot one.

The first three cases ( $\mathrm{C} 1$ to $\mathrm{C} 3$ ) concern non-uniform gaseous mixtures of $\mathrm{H}_{2} \mathrm{O}$ and $\mathrm{N}_{2}$. They consist of 2 uniform layers at different temperatures: the $\mathrm{H}_{2} \mathrm{O}$ molar fraction is 0.2 in both columns and their length is fixed to $50 \mathrm{~cm}$. In all these cases, the temperature of the cold path is $1000 \mathrm{~K}$. The temperatures of the hot layer are $1100 \mathrm{~K}(\mathrm{C} 1), 1500 \mathrm{~K}(\mathrm{C} 2)$ and $2000 \mathrm{~K}(\mathrm{C} 3)$ respectively. Results are depicted in Figures 5,7 (Spearman's coefficients $\rho_{\mathrm{SP}}$ for cases C1 and C2 - Case C3 corresponds to Figure 1), and Figures 4,6,8 (calculation of transmissivities over non-uniform paths for cases $\mathrm{C} 1, \mathrm{C} 2$ and $\mathrm{C} 3$ respectively). It can be immediately noticed that for small gradients of temperature, Spearman's coefficients for $\left(\kappa_{\eta}^{1}, \kappa_{\eta}^{2} / \kappa_{\eta}^{1}\right)$ and $\left(\kappa_{\eta}^{2}, \kappa_{\eta}^{1} / \kappa_{\eta}^{2}\right)$ are almost the same, but with opposite signs. For higher gradients, strong asymmetries appear. In these cases, the assumption of statistical independence between one spectrum and its corresponding scaling coefficient depends on the choice of the reference state and is more relevant in one direction than in the other. The choice of the hot layer as the reference (associated with $\tau_{11}$ ) was found to provide the best results over most of the narrow bands. Application of the criteria $(30,31)$ was found to suggest an index for the definition of the effective scaling function equal to 1 (viz. the hot layer is the reference) for $98.8 \%$ of the narrow bands in case $\mathrm{C} 1$ (against $99.4 \%$ if the criterion proposed in Ref. [11] is used), $99.4 \%$ in case C2 (against $100 \%$ when the direction, defined as the direction of propagation of the path $L_{1}+L_{2}$ such that the point of arrival is in the gas in the reference state, 
is chosen according to the ratios of mean absorption coefficients [11]). It reaches $100 \%$ in case C3 for both criteria. 


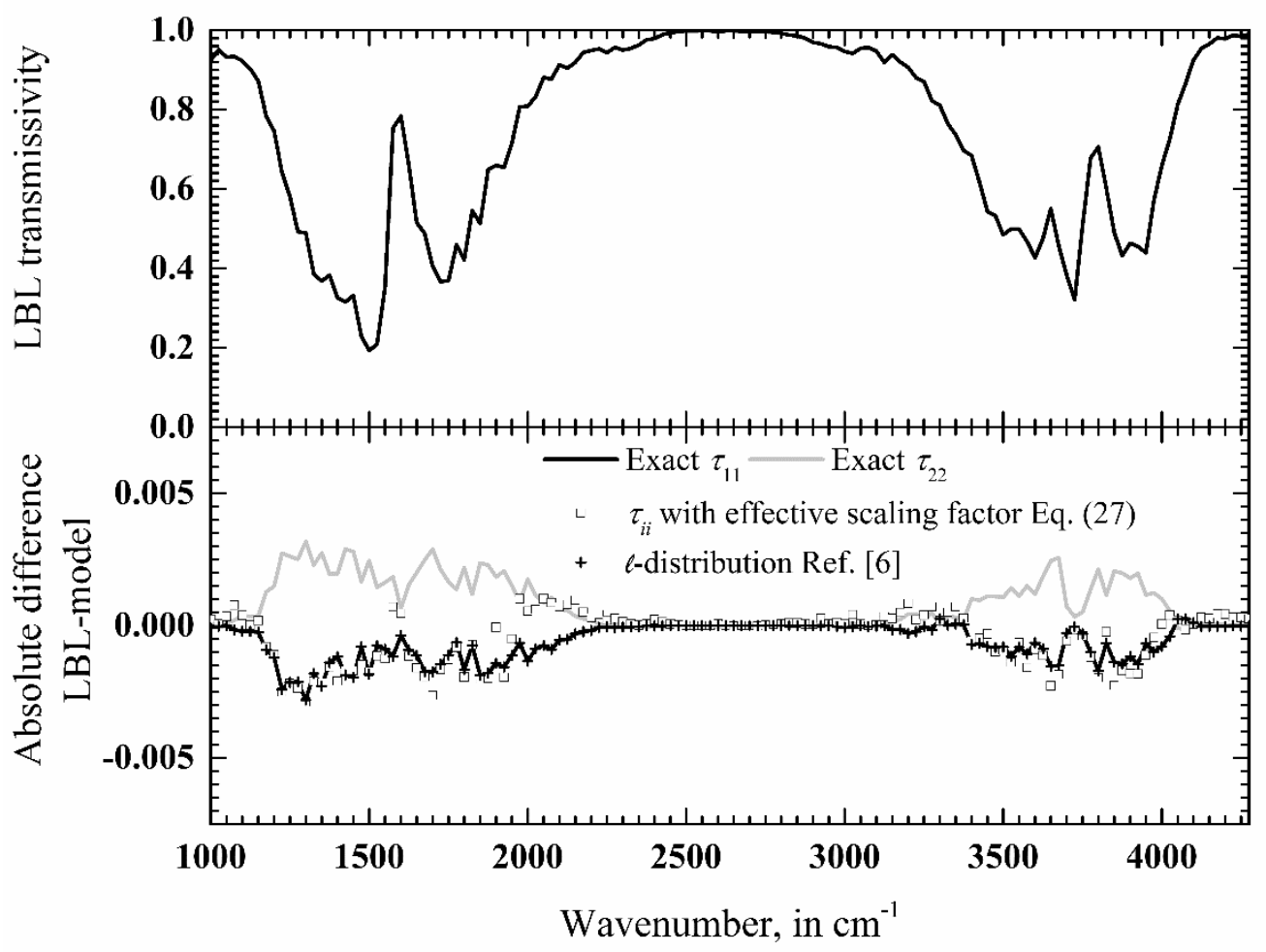

Figure 4. Transmissivities of non-uniform paths - Case C1

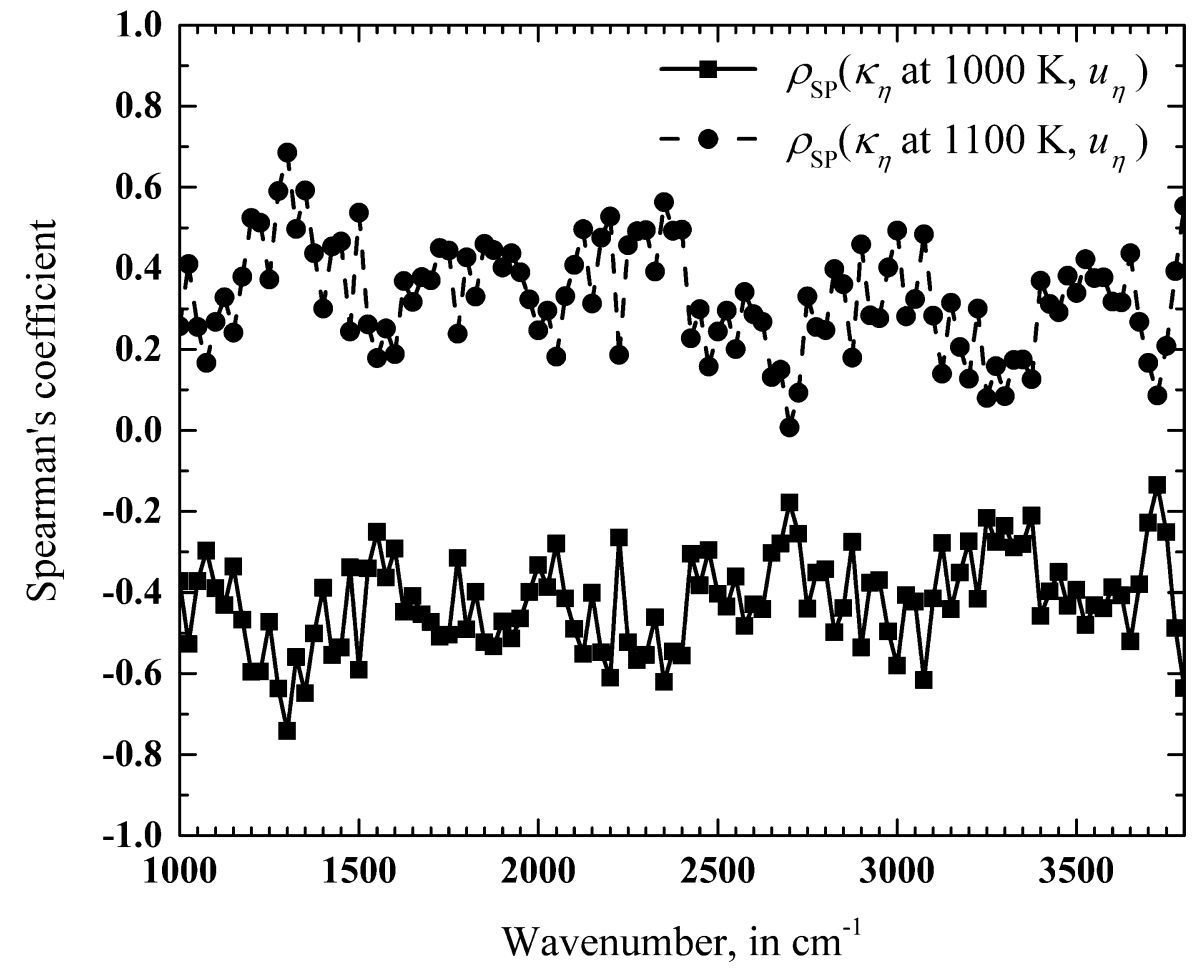

Figure 5. Narrow band Spearman's coefficients - Case C1 


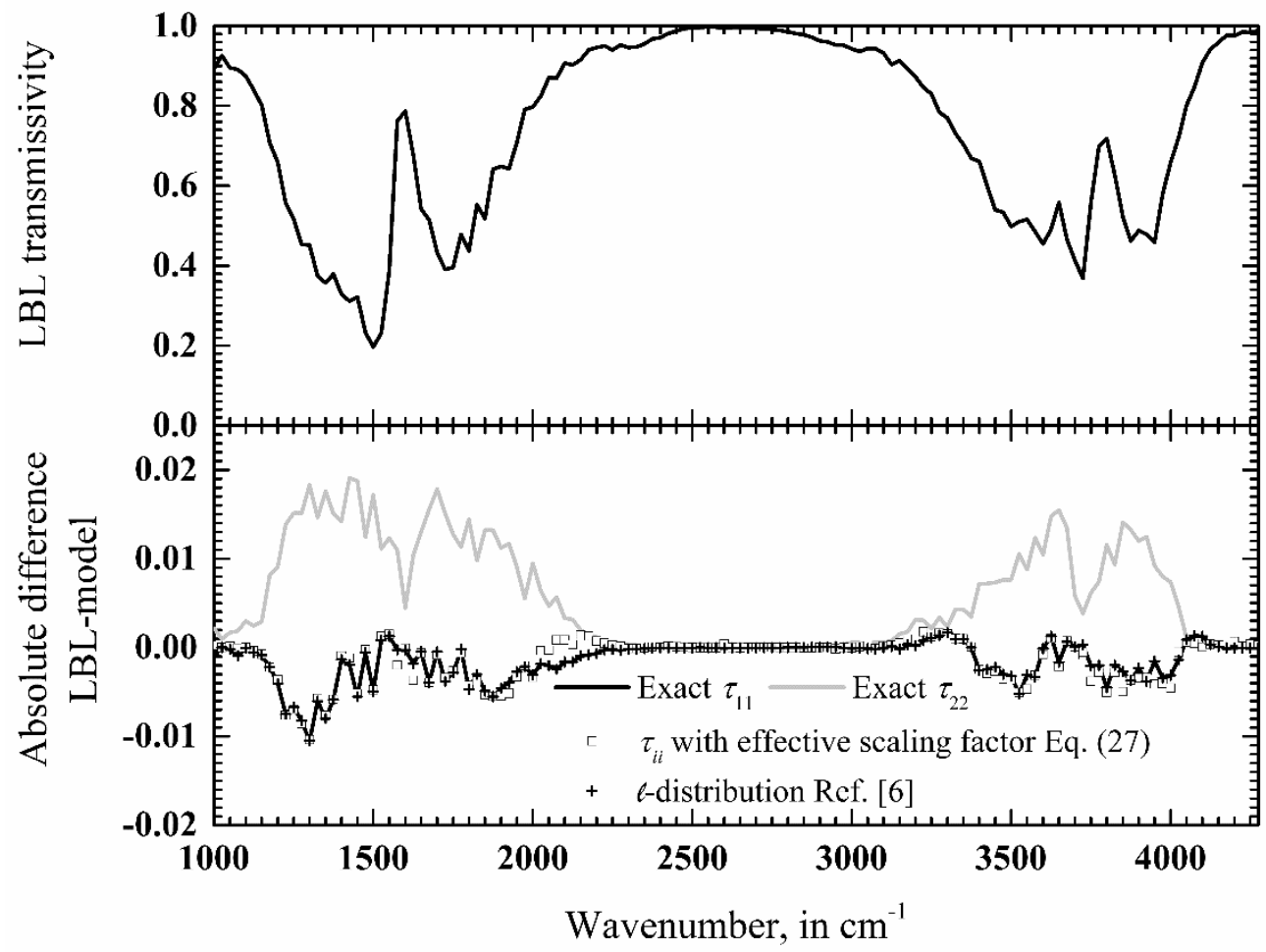

Figure 6. Transmissivities of non-uniform paths - Case C2

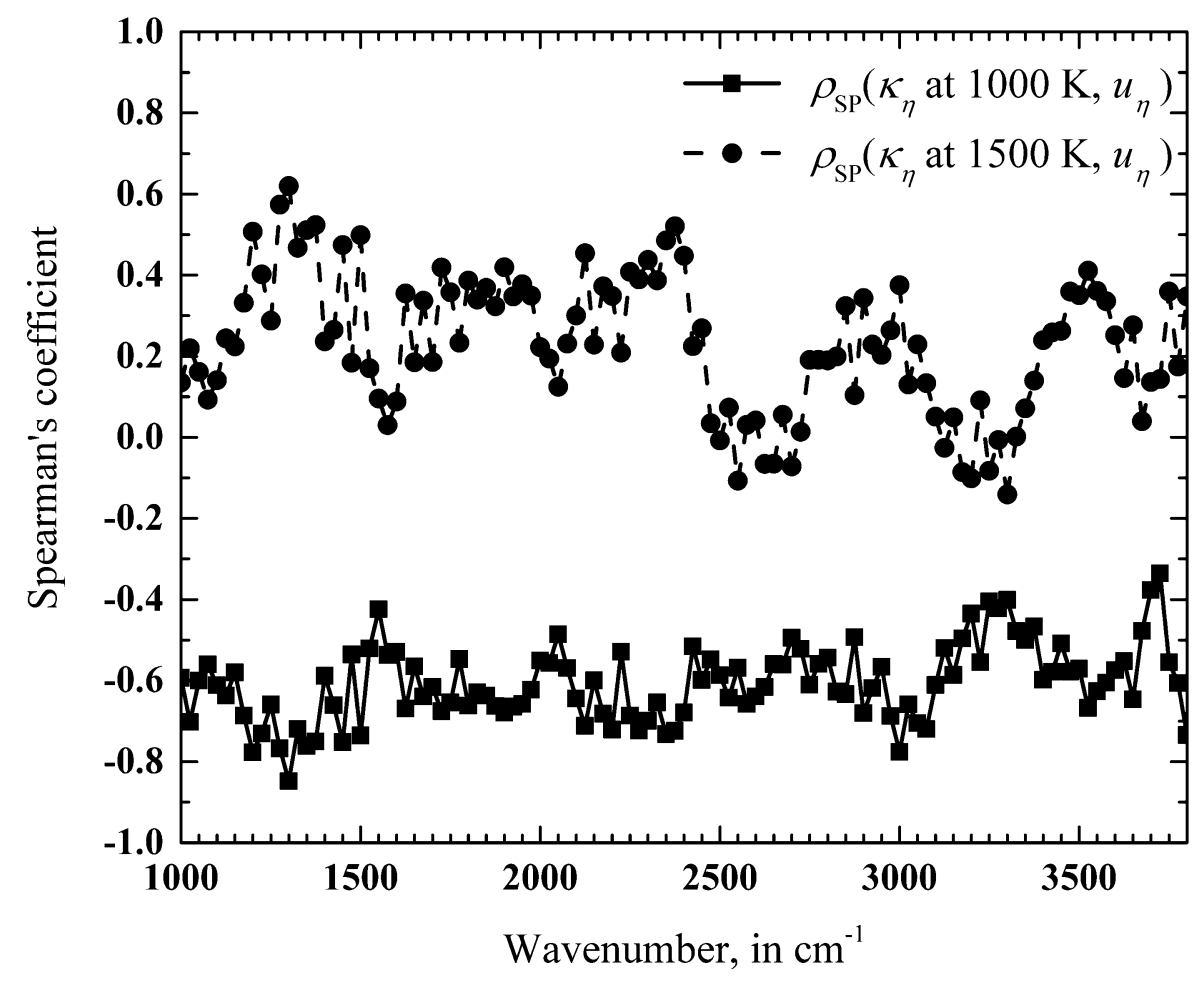

Figure 7. Narrow band Spearman's coefficients - Case C2 


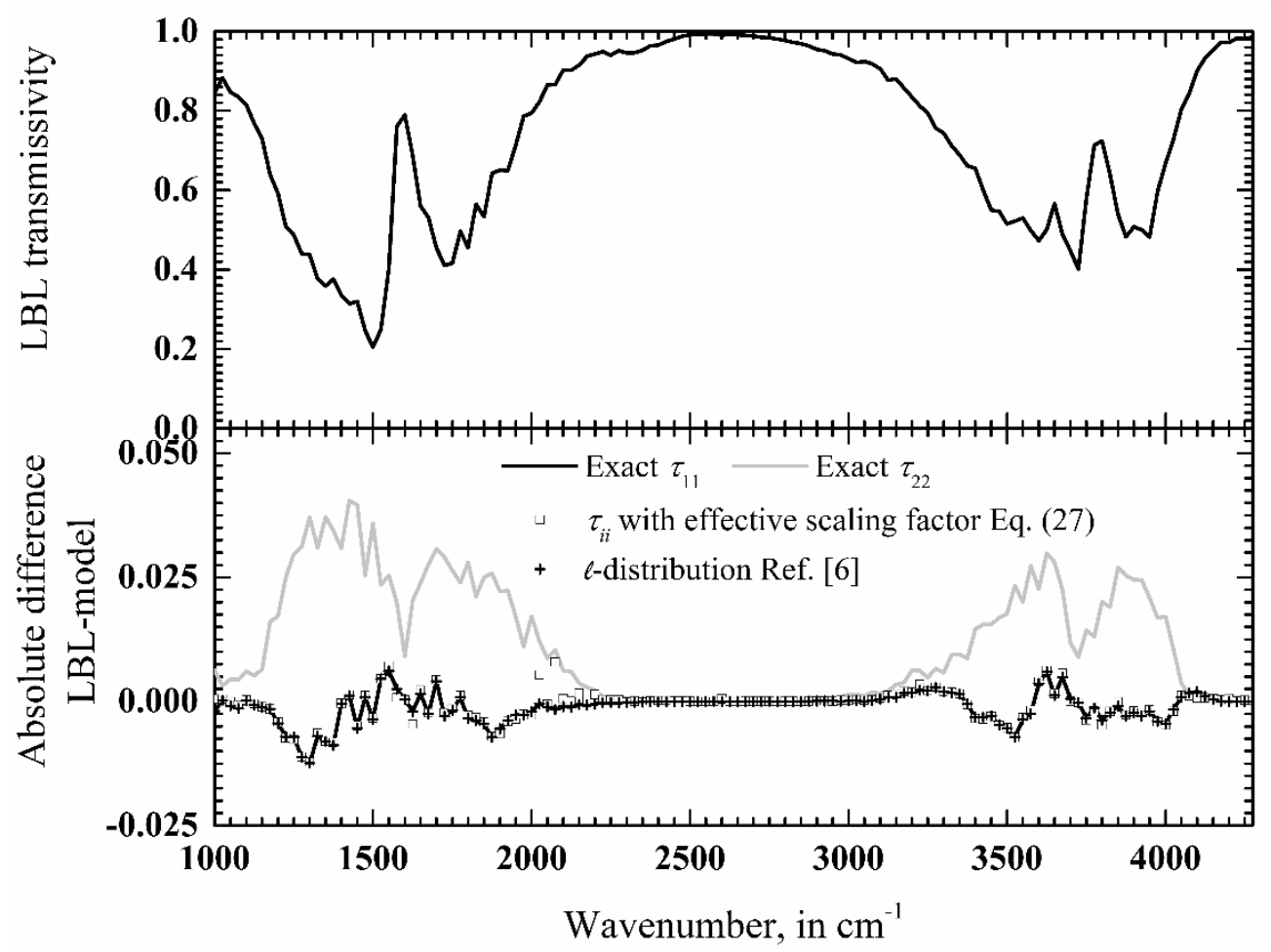

Figure 8. Transmissivities of non-uniform paths - Case C3

Case C4 was used in Ref. [5] to assess the Scaled- $k$ and Correlated- $k$ methods against LBL reference calculations. It is similar to Case C 3 but the temperature of the cold path is now $300 \mathrm{~K}$. Results are depicted in Figures 9 and 10.

Finally, the last test case, C5, was taken from Ref. [17]. It consists of two paths with different species concentrations (molar fraction of water vapor is 0.5 in the hot column, 0.05 in the cold one): the hot temperature is $1500 \mathrm{~K}$ and the length of the path is $10 \mathrm{~cm}$; the cold layer is at $500 \mathrm{~K}$ and its length is $100 \mathrm{~cm}$. This means that the product (molar fraction of absorbing species $\mathrm{x}$ length of the gas path) is the same in both layers. This test case was found in Ref. [17] to be challenging for statistical narrow band models either extended to non-uniform paths with the help of the CurtisGodson or of the Lindquist-Simmons approximations [1]. With the present method, a very acceptable accuracy is obtained as shown in Figures 11 and 12. 


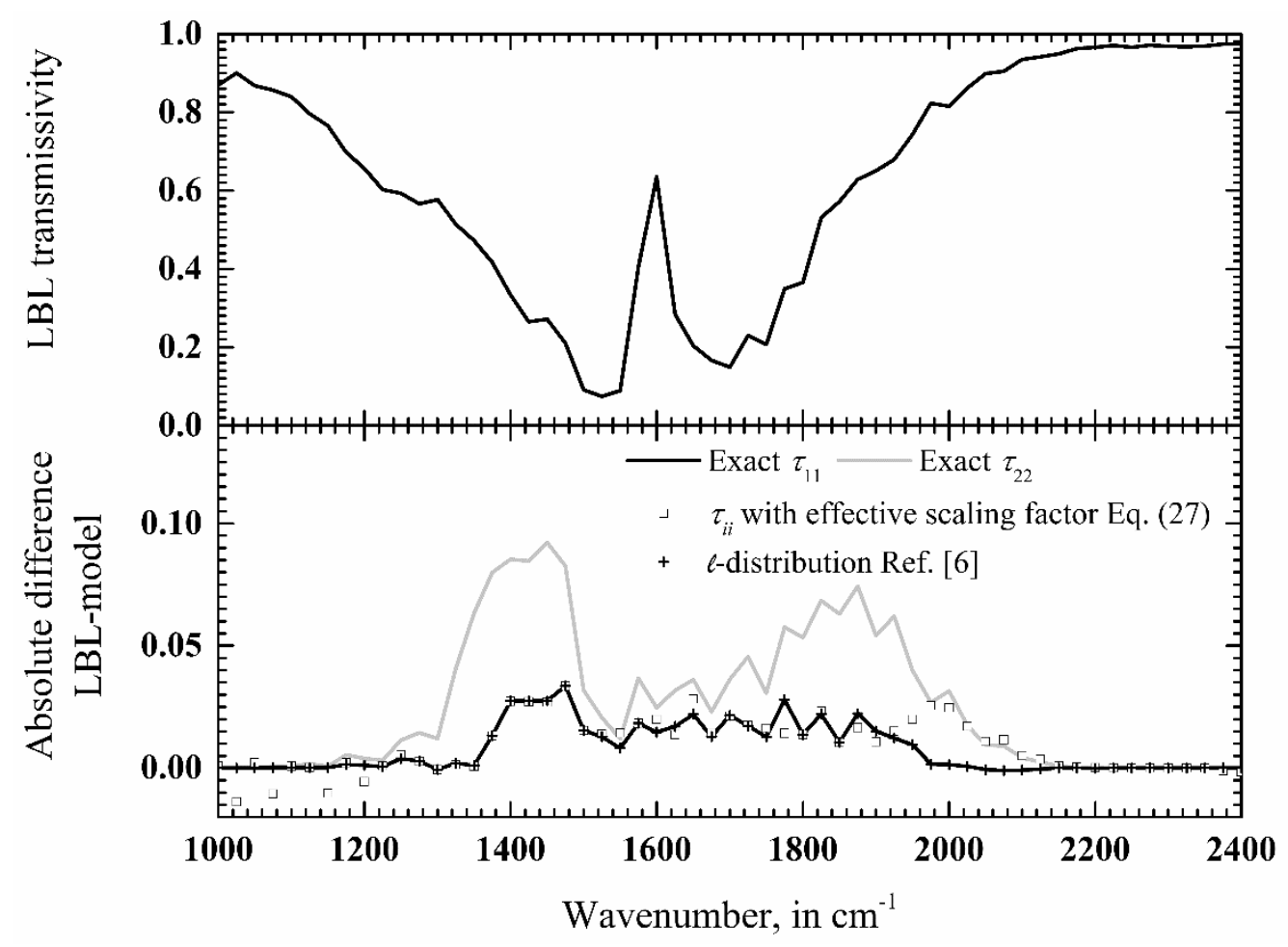

Figure 9. Transmissivities of non-uniform paths - Case C4

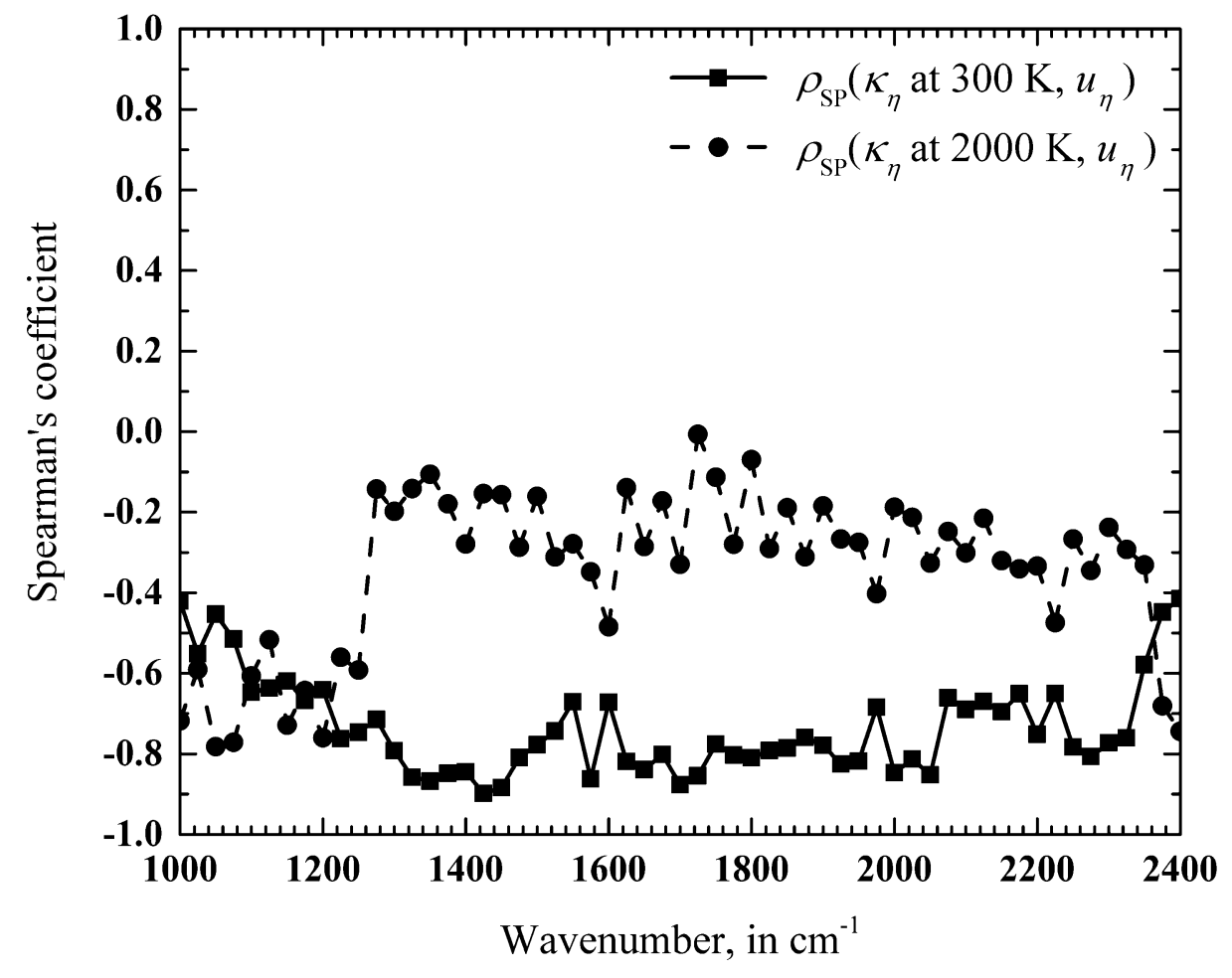

Figure 10. Narrow band Spearman's coefficients - Case C4 


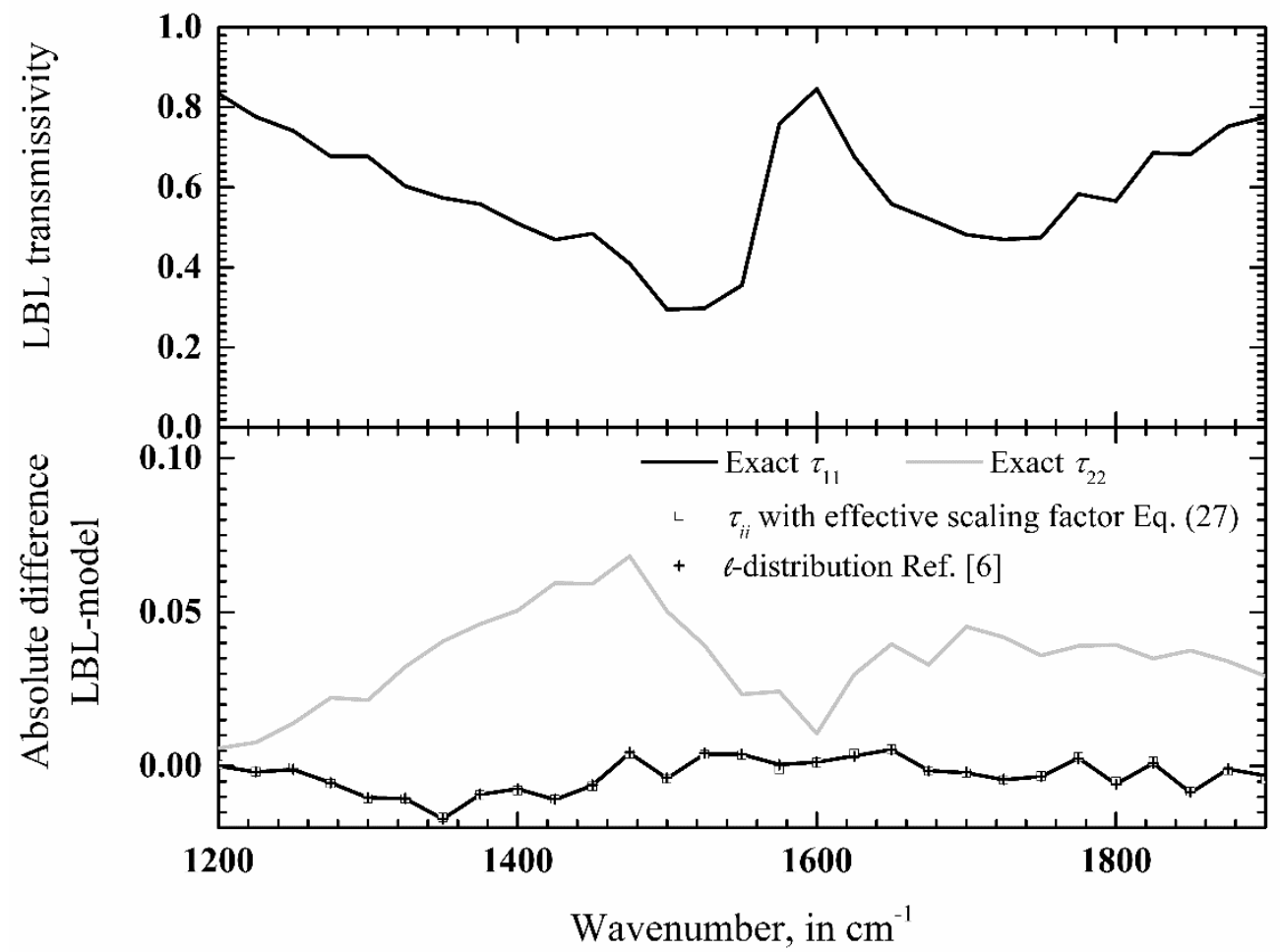

Figure 11. Transmissivities of non-uniform paths - Case C5

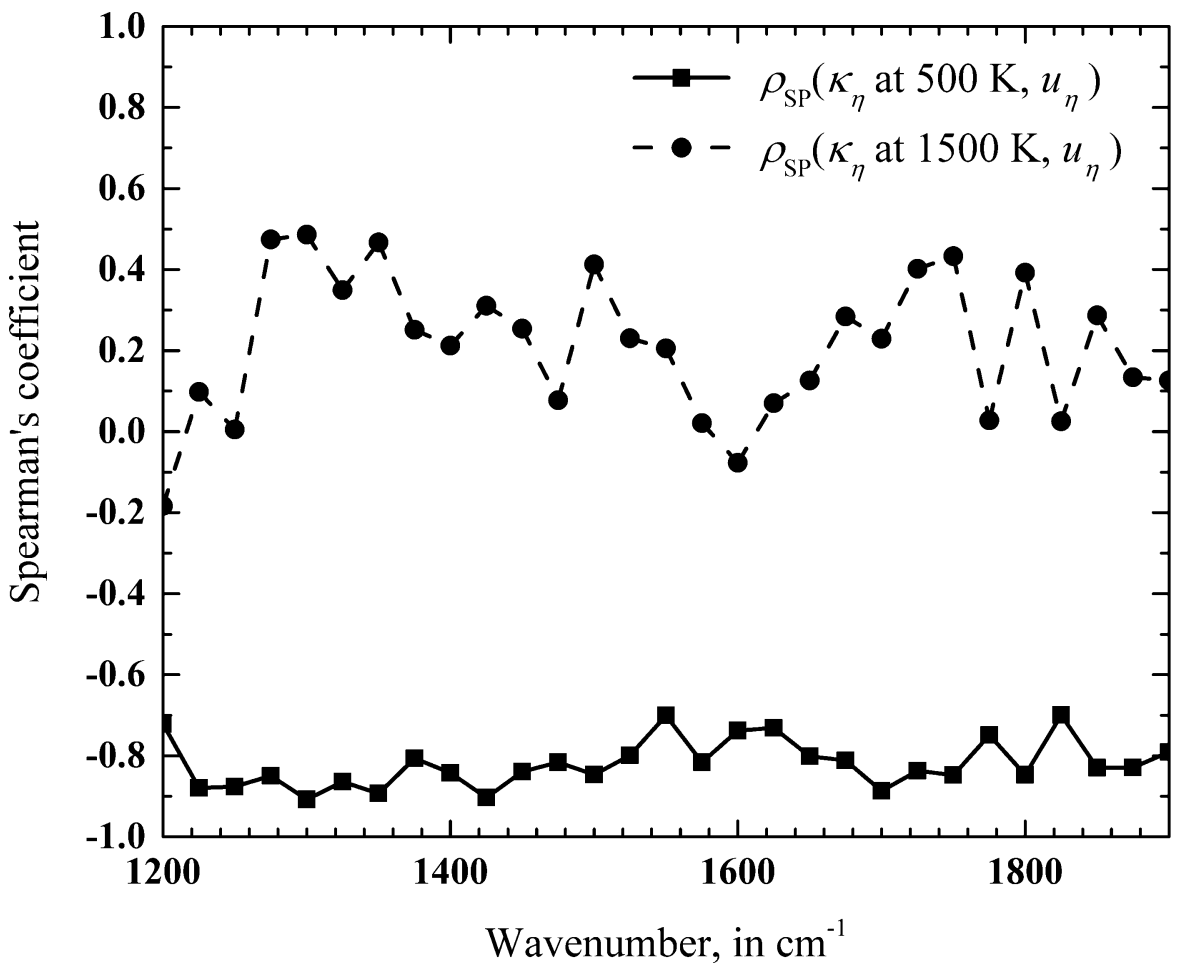

Figure 12. Narrow band Spearman's coefficients - Case C5 


\subsection{Discussion}

From the results described above, the following comments can be drawn:

$1 /$ the best solution is associated in most cases with the choice of the reference state according to the criteria $(30,31)$. This can be observed on the Figures provided in Section 3.3 by comparing the outputs of "Exact $\tau_{11}$ " with "Exact $\tau_{22}$ ". Indices in these notations represent the reference state and application of criteria $(30,31)$ suggest in most cases state " 1 " to be the reference (see the last sentence in Section 3.3). "Exact $\tau_{11}$ " is closer to exact non-uniform reference LBL calculations than "Exact $\tau_{22}$ " in almost all cases depicted in Figures $(4,6,8,9,11)$.

2/ the functional form Eq. (27) gives accurate predictions of solutions to Eq. (13) even with small quadrature orders $N$ ( $N=6$ was arbitrarily chosen in this work). When applied in non-uniform calculations, it provides results almost as precise as "exact" solutions based on a direct resolution of Eq. (13) using a bisection method.

3/ large asymmetries exist between Spearman's coefficients in the case of strong gradients of temperature. In these cases, which can be encountered in spectroscopic applications for instance, the definition of effective scaling factors requires a proper definition of the reference state. For small gradients, as in most radiative heat transfer applications for which profiles of temperatures and species concentrations are smooth and almost continuous, it was observed (case $\mathrm{C} 1$ ) that the calculations provide the same results in either directions, both in terms of Spearman's coefficients and transmissivities. In these situations, no reordering of the path is expected to improve the results significantly if a propagation scheme is used (GWN or ICE [6 ]) as in this case "reference" states are defined locally along the propagation path. However, for models based on a single reference spectrum (Scaled- $k$ method, for instance) used to scale all other states, the proper definition of the 
reference is likely to be required. This needs to be analyzed in depth and this problem can be studied by application of criteria $(30,31)$. However, the definition of a method to relate results on these couples of states to the optimum unique reference is required. These conclusions apply to the approach considered in the present work, which can be used with any model form, but also to the e-distribution method.

4/ the -distribution approach almost matches the exact solution, with a higher accuracy than the method based on Eqs. (12-13,27-28). However, this second technique provides results very close to the exact solution and has the advantage of being applicable with any uniform model (LBL or $k$-distribution, for instance).

5/ The explicit method based on Eqs. $(13,27-28)$ together with a LBL or $k$-distribution model requires the evaluation of two integrals: one over wavenumbers (LBL) or $k$-values ( $k$-distribution); the other one over $u$, i.e. Eq. (27). This makes the -distribution approach, which requires no integral, more computationally efficient than these methods. However, many existing codes already use a $k$-distribution technique: for all of them, Scaled methods based on effective scaling functions (which can be constructed using the scheme described in the present work) can be considered as relevant alternatives to the widely used Correlated- $k$ approximation. However, although the use of the explicit formula Eq. (27) is more computationally efficient than a direct iterative solving of Eq. (13), the method remains more expensive than a C- $k$ approximation. Nevertheless, as a counterpart and following the existing literature on models based on effective scaling factors $[1,5,6,11]$, a higher accuracy of the non-uniform approximation can be expected with the scaled approach than with the correlated one. 


\section{CONCLUSION}

Non-uniform methods based on effective scaling factors were among the first to appear in the history of approximate models for the radiative properties of gases. The idea introduced by Godson in 1953 was rediscovered several times. All these works have reached the same conclusion: this approach is more accurate than other, more established, techniques such as Correlated- $k$ for instance. One reason that may explain the apparent lack of interest for such methods, not often encountered in the literature, is the absence of explicit ways to evaluate effective scaling factors. The aim of the present paper was to suggest a solution to this issue by proposing an analytical formula for the calculation of effective scaling factors, together with an analysis of the conditions on gas spectra and spectral scaling coefficients for this formulation to be acceptable. This formula, Eq. (27), is the core result of the present paper.

The other main findings of the present work are:

$1 /$ the origin of asymmetries encountered in models based on effective scaling factors, which were shown to be closely related to the assumption of statistical independence between gas spectra and spectral scaling coefficients. In highly non-uniform two-cell problems, it was found that only one choice for the gas reference spectrum is possible to ensure this assumption to be reasonable;

$2 /$ the definition of the parameters that appear in effective scaling functions requires an optimization process, fully detailed in the paper;

3/ after optimization, the use of the explicit formula Eq. (27) provides results almost as accurate as a direct approximation of scaling factors by an iterative solving of the implicit equation Eq. (13). The use of this explicit formula is less computationally demanding than any iterative process (bisection, Newton's method, etc). 
The main value of the results described in this paper is to open new possibilities for application together with all existing codes of gas radiation. This is because non-uniform approximations based on effective scaling factors can be applied to any model form. This fact was discussed, for instance, by Young in his recent book.

\begin{abstract}
AKNOWLEDGEMENTS
This work has been supported by the Programme National de Télédétection Spatiale (PNTS, http://www.insu.cnrs.fr/pnts grant $N^{\circ}$ PNTS-2017-04).
\end{abstract}




\section{REFERENCES}

[1] YOUNG S.J. Band model theory of radiation transport, The Aerospace Press, 2013. ISBN: 978$1-884989-25-4$.

[2] LACIS A., WANG W.-C., HANSEN J.E., Correlated k-distribution method for radiative transfer in climate models: application to effect of cirrus clouds on climate, NASA Goddard Space Flight Center, $4^{\text {th }}$ NASA Weather and Climate Program Sci. Rev. 1979:309-314.

[3] WEINREB M.P., NEUENDORFFER A.C. Method to apply homogeneous-path transmittance models to inhomogeneous atmospheres, J. Atm. Sci. 1973;30:662-666.

[4] GORDLEY L.L., RUSSELL III J.M. Rapid inversion of limb radiance data using an emissivity growth approximation, App. Opt. 1981;20:807-813.

[5] MODEST MF. Narrow-band and full-spectrum $k$-distributions for radiative heat transfer correlated- $k$ vs. scaling approximation, JQSRT 2003;76:69-83.

[6] ANDRE F. The -distribution method for modeling non-gray absorption in uniform and nonuniform gaseous media, JQSRT 2016;179:19-32.

[7] SOLOVJOV V.P., ANDRE F., LEMONNIER D., WEBB B.W. The Scaled SLW model of gas radiation in non-uniform media, ICHMT International Symposium on Advances in Computational Heat Transfer CHT-17, Napoli, 2017.

[8] GODSON W.L. The evaluation of infra-red radiative fluxes due to atmospheric water vapor, Q. J. R. Met. Soc. 1953;79:367-379.

[9] SCHILLING R., SONG R., VONDRACEK Z., Bernstein functions: Theory and Applications, Studies in Mathematics, 37, de Gruyter, Berlin, 2010. 
[10] HERING C., HOFERT M., MAI J.-F., SCHERE M., Constructing hierarchical Archimedean copulas with Levy subordinators, J. Multivariate Analysis 2010;101:1428-1433.

[11] ANDRE F., An analysis of the symmetry issue in the $\ell$-distribution method of gas radiation in non-uniform gaseous media, JQSRT 2017;190:78-87.

[12] NELSEN R.B. An introduction to Copulas - Second Edition, Springer series in statistics, Springer, 2006.

[13] JOE H. Multivariate models and dependence concepts, Monograph on statistics and applied probability 73, Springer Science+Business Media Dordrecht 1997.

[14] ANDRE F, HOU L, ROGER M, VAILLON R. The multispectral gas radiation modeling: a new theoretical framework based on a multidimensional approach to $k$-distribution methods, JQSRT 2014;147:178-195.

[15] ROTHMAN L.S., GORDON L.E., BARBER R.J., DOTHE H., GAMACHE R.R., GOLDMAN A., PEREVALOV V.I., TASHKUN S.A., TENNYSON J., HITEMP, the hightemperature molecular spectroscopic database, JQSRT 2010;111:2139-2150.

[16] Numerical recipes in Fortran 77: the art of scientific computing. Cambridge: Cambridge University Press; 1992.

[17] SOUFIANI A., HARTMANN J.M., TAINE J. Validity of band model calculations for $\mathrm{CO}_{2}$ and $\mathrm{H}_{2} \mathrm{O}$ applied to radiative properties and conductive-radiative transfer, JQSRT 1985;33:243257. 


\section{APPENDIX}

\section{A.1. Theoretical justification of Eqs. $(8,9)$ in the independent case}

Let $f$ be a bivariate function of a spectral absorption coefficient $\kappa_{\eta}$ and a scaling coefficient $u_{\eta}$. It was shown in reference [14] that the mean value of function $f$ over a narrow band $\Delta \eta$ can be written in distribution form as:

$$
f^{\Delta \eta}=\frac{1}{\Delta \eta} \cdot \int_{\Delta \eta} f\left(\kappa_{\eta}, u_{\eta}\right) d \eta=\int_{0}^{+\infty} \int_{0}^{+\infty} f(k, u) d M(k, u)
$$

where $M(k, u)$ is the joint distribution function of $\kappa_{\eta}$ and $u_{\eta}$ :

$$
M(k, u)=\mathbb{P}\left(\kappa_{\eta}<k, u_{\eta}<u\right)=\frac{1}{\Delta \eta} \cdot \int_{\Delta \eta} H\left(k-\kappa_{\eta}\right) \cdot H\left(u-u_{\eta}\right) d \eta
$$

If $\kappa_{\eta}$ and $u_{\eta}$ are statistically independent, then this joint distribution can be written as the product

of the distribution of each variable, i.e. $M(k, u)=g(k) \cdot F(u)$, to yield:

$$
f^{\Delta \eta}=\frac{1}{\Delta \eta} \cdot \int_{\Delta \eta} f\left(\kappa_{\eta}, u_{\eta}\right) d \eta=\int_{0}^{+\infty} \int_{0}^{+\infty} f(k, u) d g(k) d F(u)
$$

Application of (A.3) to uniform and non-uniform transmissivities provides Eqs. (8) and (9).

Indeed, if we replace for instance $f$ by $\exp \left(-\kappa_{\eta}^{1} u_{\eta} L\right)$ we obtain directly:

$$
\tau_{2}^{\Delta \eta}\left(L_{2}\right)=\frac{1}{\Delta \eta} \cdot \int_{\Delta \eta} \exp \left(-\kappa_{\eta}^{1} u_{\eta} L_{2}\right) d \eta=\int_{0}^{+\infty}\left[\int_{0}^{+\infty} \exp \left(-k u L_{2}\right) d g(k)\right] d F(u)=\int_{0}^{+\infty} \tau_{1}^{\Delta \eta}\left(u L_{2}\right) d F(u)
$$


which is Eq. (8). A similar derivation provides Eq. (9).

\section{A.2. Connections with Godson's method}

Let us now show up some connections between the approach described in the paper and Godson's method [1,8]. For this purpose, we start by rewriting Eq. (A.4) as:

$$
\tau_{2}^{\Delta \eta}\left(L_{2}\right)=\int_{0}^{1} \tau_{1}^{\Delta \eta}\left[F^{-1}(\xi) L_{2}\right] d \xi
$$

For a given length $L_{0}$, application of the mean value theorem shows that one can find a particular value $\xi_{0}$ of $\xi$ such that:

$$
\tau_{2}^{\Delta \eta}\left(L_{0}\right)=\int_{0}^{1} \tau_{1}^{\Delta \eta}\left[F^{-1}(\xi) L_{2}\right] d \xi=\tau_{1}^{\Delta \eta}\left[F^{-1}\left(\xi_{0}\right) L_{0}\right]
$$

Different values of $L_{0}$ may provide distinct values of $\xi_{0}$. Accordingly, by choosing a large number of values of $\xi_{0}$ one can define pointwise a function $X$ of $L_{0}$ such that for any $L_{0}, X\left(L_{0}\right)=\xi_{0}$ where $\xi_{0}$ corresponds to the solution of Eq. (A.6). Once combined with $F^{-1}$ we thus obtain a function of $L_{0}$ such that for any length $L_{0}$ :

$$
\tau_{2}^{\Delta \eta}\left(L_{0}\right)=\tau_{1}^{\Delta \eta}\left(F^{-1}\left[X\left(L_{0}\right)\right] L_{0}\right)
$$

Function $F^{-1}[X(L)]$ corresponds to function $u(L)$ in Eq. (13).

The same method can be applied in the non-uniform case. But instead of the definition of a function of a single variable as in uniform situations, we receive in this case a function that both depends on $L_{1}$ and $L_{2}$ and which takes the following form: $F^{-1}\left[X^{\prime}\left(L_{1}, L_{2}\right)\right]$. By definition, this function 
returns values between the minimum and the maximum of the spectral scaling coefficient over the narrow band. Accordingly, if we further assume that the interval of $u$ values is small, then one can use the following approximation:

$$
F^{-1}\left[X^{\prime}\left(L_{1}, L_{2}\right)\right] \approx F^{-1}\left[X^{\prime}\left(L_{1}=0, L_{2}\right)\right]=F^{-1}\left[X\left(L_{2}\right)\right]
$$

This approximation provides Eq. (12).

This shows that the method presented in the paper can be written in the same form as Godson's, EGA or scaled- $k$ techniques. It must be however noticed that in the truly independent case, formula Eq. (9) provides the exact solution whereas Eq. (13) is only approximate due to (A.8). On the other hand, for weakly dependent situations, errors may appear if Eq. (8) is used to represent the uniform path. Eq. (13) is in this case exact.

Stronger connections between the two approaches can be established if one assumes that, in the weakly dependent case, it is possible to define a subrange of values of $k$, written $D$, such that:

$$
\left\{\begin{array}{l}
\tau_{2}^{\Delta \eta}\left(L_{2}\right)=\int_{0}^{1} \tau_{1}^{\Delta \eta}\left[F_{D}^{-1}(\xi) L_{2}\right] d \xi \\
F_{D}(u)=\frac{\int_{\left\{\eta: \kappa_{\eta} \in D\right\}} H\left(u-u_{\eta}\right) d \eta}{\int_{\left\{\eta: \kappa_{\eta} \in D\right\}} d \eta} d \eta
\end{array}\right.
$$

Then replacing $\xi$ by $F(u)$ we obtain:

$$
\tau_{2}^{\Delta \eta}\left(L_{2}\right)=\int_{0}^{+\infty} \tau_{1}^{\Delta \eta}\left[T_{D}(u) L_{2}\right] d F(u), T_{D}(u)=F_{D}^{-1}[F(u)]
$$


(A.10) takes a form similar to Eq. (8) but, in this case, variable $u$ does not appear directly but is modulated by some function $T_{D}$. By definition, this function $T_{D}: 1 /$ preserves the moments of the absorption coefficient and ensures the transition from Eq. (A.9) to Eq. (13) to be exact; 2/ values of $T_{D}(u)=F_{D}^{-1}[F(u)]$ are in a bounded interval included inside the domain of variation of the spectral scaling coefficient over the narrow band i.e. $\left[u_{\min } ; u_{\max }\right]$. The size of the image of $\left[u_{\min } ; u_{\max }\right]$ through $T_{D}$ is thus smaller than $\left[u_{\min } ; u_{\max }\right]$ and, as a consequence, spectra are "more scaled" over $D$ than over $\Delta \eta$ : this connects this formulation directly with the assumptions made in Godson's method (see the last sentence above Eq. (A.8)). There are undoubtedly strong connections between the domain $D$ introduced here and the mean absorption coefficient $\mu_{S}$ used in the paper, but this analysis is kept as future work.

\section{A.3. Justification of Eq. (11)}

Spearman's coefficient defined as $[12,13]$ :

$$
\rho_{\mathrm{SP}}\left(\kappa_{\eta}^{1}, u_{\eta}\right)=12 \cdot \frac{1}{\Delta \eta} \cdot \int_{\Delta \eta} g(k) F(u) d M(k, u)-3
$$

can be written equivalently, using Eq. (A.1), as:

$$
\rho_{\mathrm{SP}}\left(\kappa_{\eta}^{1}, u_{\eta}\right)=12 \cdot \frac{1}{\Delta \eta} \cdot \int_{\Delta \eta} g\left(\kappa_{\eta}^{1}\right) F\left(u_{\eta}\right) d \eta-3
$$

which is Eq. (11). It suffices to apply (A.1) to the function $f(k, u)=g(k) F(u)$. 